\title{
Anabases
}

ANABASES Traditions et réceptions de l'Antiquité

27 | 2018

Varia

\section{Restituer l'emplacement des statues dans les édifices d'Herculanum ? Problèmes de méthode}

\section{Emmanuelle Rosso Caponio}

\section{(2) OpenEdition}

1 Journals

Édition électronique

URL : http://journals.openedition.org/anabases/6756

DOI : 10.4000/anabases.6756

ISSN : 2256-9421

Éditeur

E.R.A.S.M.E.

\section{Édition imprimée}

Date de publication : 1 avril 2018

Pagination : 67-84

ISSN : 1774-4296

\section{Référence électronique}

Emmanuelle Rosso Caponio, «Restituer l'emplacement des statues dans les édifices d'Herculanum?

Problèmes de méthode », Anabases [En ligne], 27 | 2018, mis en ligne le 01 avril 2020, consulté le 20 janvier 2021. URL : http://journals.openedition.org/anabases/6756 ; DOI : https://doi.org/10.4000/ anabases.6756 


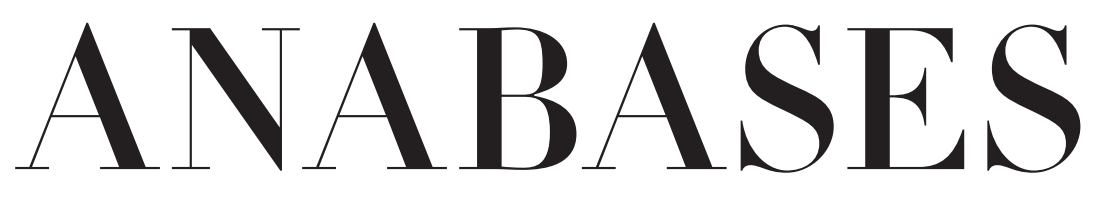

Traditions et Réceptions de l'Antiquité

$$
\begin{aligned}
& \text { No27 } \\
& 2018
\end{aligned}
$$

E.R.A.S.M.E.

Université Toulouse - Jean Jaurès 



\section{Sommaire}

$\mathrm{N}^{\circ} 27-2018$

\section{Historiographie et identités culturelles}

\section{Sébastien Cazalas}

“ et pour ce recite Valere... ». La place de l'Antiquité dans l'arsenal

intellectuel d'un grand prélat français du XVe siècle :

Jean Juvénal des Ursins (I388-I473) . . . . . . . . . . . . . . . . . . . II

Loic Marcou

La réception de l'Antiquité dans l'œuvre de la femme de lettres grecque

Rhéa Galanaki. . . . . . . . . . . . . . . . . . . . . .

\section{Traditions du patrimoine antique}

« Restituer Herculanum II. Des archives de fouilles aux restitutions 3D

Alexandra DARDENAY

Introduction : restituer l'espace domestique à Herculanum

grâce aux outils informatiques de reconstruction virtuelle ;

enjeux et problèmes. . . . . . . . . . . . . . . 4

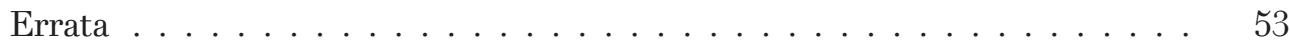

Carla Marotta

L’utilisation des données archivistiques dans l'enquête archéologique : nouvelles découvertes sur la maison d'Argos . . . . . . . . . . . . . . .

Emmanuelle Rosso

Restituer l'emplacement des statues dans les édifices d'Herculanum?

Problèmes de méthode . . . . . . . . . . . . . . . 7I 
Hélène Eristov, Marie-Laure Maraval

Restituer les décors perdus de la maison de Neptune et d'Amphitrite :

enquête, méthodes, résultats . . . . . . . . . . . . 9 9I

Maud Mulliez

Restauration numérique des peintures murales de la maison

de Neptune et Amphitrite à Herculanum : de l'expérimentation

matérielle à l'intégration des données dans un modèle 3D . . . . . . . Io3

\section{Archéologie des savoirs}

Nicolas SIRON

L'histoire de Philippidès d'Hérodote à Lucien.

Une incursion dans l'atelier du mythe . . . . . . . . . . . . . . . . Iog

Constantin Raios

Le coracin (xo@axîvos) du lac de Tibériade (F. Josèphe, B.J. III, 520) . . .

\section{Actualités et débats}

Claude Azıza

Antiquités parallèles (8). Le syndrome du sein droit . . . . . . . . . . . . I6I

Elisabeth DÉcultot

Winckelmann. Moderne Antike / Winckelmann. Modern Antiquity.

Exposition, Weimar, Neues Museum, 7 avril-2 juillet 20I7 . . . . . . . I67

\section{Lire, relire la bibliothèque des sciences de l'Antiquité}

Cyrielle LANDrea

Jérôme Carcopino et la mémoire perdue

de M. Valerius Messalla Corvinus (cos. 3г av. J.-C.) . . . . . . . . . . . . $\quad$ I77

Jérôme CARCopino

Notes biographiques sur M. Valerius Messala Corvinus . . . . . . . . . I I85

\section{L'atelier de l'histoire : chantiers historiographiques}

L'Atelier des doctorants (coordonné par Adeline Grand-Clément) (15)

Jonathan Pérez Mostazo

Cantabri aut vascones. La reception de la Antigüedad

en la cultura histórica vasca del siglo XIX . . . . . . . . . . . . . . . . . 209 


\section{Comptes rendus}

William H.F. Altman (éd.)

Brill's Companion to the Reception of Cicero (A. A. Raschieri). . . . . . . . 2 2I9

Anthony A. Barrett, Elaine Fantham, John C. Yardley

The Emperor Nero. A Guide to the Ancient Sources (É. Deniaux) . . . . . . 22I

Pascale Barthélémy et Violaine sébillotte cuchet (éd.)

Clio. Femmes, Genre, Histoire $\mathrm{n}^{\circ} 43$ : Citoyennetés (M. L. Napolitano) . . . 222

Frédéric Colin, Olivier Huck, Sylvie Vanseveren (éd.)

Interpretatio. Traduire l'altérité culturelle dans les civilisations

de l'Antiquité (Cl. Joncheray) . . . . . . . . . . . . . . . . . . . . 224

Angus Fletcher

Comic Democracies. From Ancient Athens

to the American Republic (A. de Crémoux). . . . . . . . . . . . . . . . . . 226

David Hernández de la Fuente

El despertar del alma. Dioniso y Ariadna: mito y misterio (A. Iriarte) . . . . 228

Ted Kaizer (éd.)

Religion, Society and Culture at Dura-Europos (P. G. Michelotto) . . . . . 229

Jacques Le Goff, Jean-Pierre Vernant

Dialogue sur l'histoire. Entretiens avec Emmanuel Laurentin (C. Lucci). . 23I

Justine Mac Connell, Edith Hall

Ancient Greek Myth in World Fiction since ig8g (A. de Crémoux) . . . . . . 233

Angelo Mazzocco, Marc Laureys (éd.)

ANew Sense of the Past. The Scholarship

of Biondo Flavio (I3g2-I463) (C. Lucci). . . . . . . . . . . . . . . . . . . . . . 234

Seth L. Schein

Homeric Epic and Its Reception. Interpretative Essays (S. Sistac) . . . . . . 236

Arbogast SснмiтT

Wie aufgeklärt ist die Vernunft der Aufklärung?

Eine Kritik aus aristotelischer Sicht (L. Calvié) . . . . . . . . . . . . . . . . 238

Richard Sorabu (éd.)

Aristotle Re-Interpreted. New Findings on Seven Hundred Years

of the Ancient Commentators (P. Butti de Lima) . . . . . . . . . . . . . . . 239

Laura SwiFT

Greek Tragedy. Themes and Contexts (G. Aujac) . . . . . . . . . . . . . . 24

Zara Martirosova TorLone

Vergil in Russia. National Identity and Classical Reception

(D. Millet-Gérard) . . . . . . . . . . . . . . . . . . 243 
Jürgen von UNGERN-STERNBERG

Les chers ennemis. Deutsche und französische

Altertumswissenschaftler in Rivalität und Zusammenarbeit (L. Calvié) . . 2444

Robert W. WALLACE

Reconstructing Damon: Music, Wisdom Teaching, and Politics

in Perikles'Athens (A. Brancacci) . . . . . . . . . . . . . . . . . . . . . 246

T. P. Wiseman

The Roman Audience : Classical Literature as Social History (C. Landrea) 249

Résumés . . . . . . . . . . . . . . . . . . . . . . . . . . 253

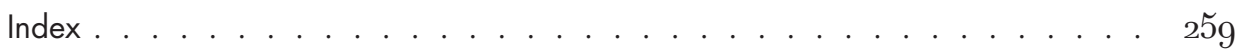




\section{Traditions}

\section{du patrimoine antique}

Restituer Herculanum II.

Des archives de fouilles aux restitutions 3D 



\section{Restituer l'emplacement des statues dans les édifices d'Herculanum ? Problèmes de méthode'}

Emmanuelle Rosso

'étude des décors en contexte qui est au cœur du projet VESUVIA ne pouvait évacuer toute enquête sur la décoration statuaire des espaces domestiques d'Herculanum, parallèlement à celle qui est menée pour les décors peints. Les difficultés que soulève une telle entreprise sont bien évidemment communes à tous les types de décors et ont été déjà abondamment évoquées. Toutefois, les œuvres sculptées posent des problèmes spécifiques qui tiennent à leur nature d'objets relevant du mobilier, pour lesquels aucun lien structurel ne peut donc être établi avec l'environnement architectural demeuré en place, quelle que soit la majesté des décors.

Quoique plusieurs maisons d'Herculanum doivent leur nom conventionnel à une sculpture remarquable trouvée dans l'une de leurs pièces - Casa dei Cervi, Casa del rilievo di Telefo ou Casa dell'Erma di bronzo par exemple - certaines images abondamment diffusées sont trompeuses : dans la plupart des cas, les statues n'ont pas été découvertes à l'emplacement de leur exposition originelle ${ }^{2}$

1 Je tiens à remercier chaleureusement Alexandra Dardenay et Adeline GrandClément pour leur invitation à contribuer à ce volume. Mes remerciements vont également à Nicolas Monteix, qui m’a communiqué des données inédites sur les circonstances de découverte de certains fragments sculptés (cf. notamment la fig. 3) et qui a réalisé les figures 6 et 8 à 10 .

2 Pour Pompéi, il est acquis désormais que les récupérateurs de matériaux ont œuvré peu de temps après l'éruption de 79 et dépouillé les espaces publics de leurs ornements de bronze mais aussi de marbre, et jusqu'aux placages : W. VAn ANDringa, 
et leurs moulages ne sont pas nécessairement exposés aujourd'hui sur le lieu exact de leur découverte. Le flux pyroclastique qui a dévasté Herculanum a entraîné un arrachement et une dispersion des objets à une échelle et sur des distances parfois tout à fait étonnantes. Ainsi, alors que le corpus des sculptures est d'une exceptionnelle richesse par la variété des formats, des matériaux et des types iconographiques attestés, pour la plupart des édifices privés, la nature de la documentation qui nous est parvenue est d'interprétation très délicate. En effet, outre les conditions d'ensevelissement du site, il faut prendre en compte également celles, plus nombreuses encore, qui tiennent à la mise au jour des structures et des objets. En d'autres termes, le déplacement antique puis la spoliation moderne des sculptures constituent deux obstacles majeurs à la restitution de leur emplacement originel ; d'où la nécessité non seulement d'une collecte, mais aussi d'une analyse critique de nos sources. Après une présentation synthétique des principaux écueils méthodologiques auxquels se heurte un rattachement des sculptures d'Herculanum à leur emplacement d'origine, je m'attarderai sur deux études de cas qui me paraissent significatives.

\section{Des contextes de découverte le plus souvent inconnus}

Il importe de rappeler en premier lieu que les plus anciennes explorations programmées ne visaient pas à connaître la topographie ou l'urbanisme de la ville, mais bien à récupérer des bronzes et des marbres dignes de figurer dans les collections royales : un très grand nombre de sculptures ont été “ prélevées » par les cavamonti du XVIII ${ }^{\mathrm{e}}$ siècle ${ }^{3}$ à cette fin. Après avoir visité le musée du Palais Royal de Portici, J. J. Winckelmann affirma qu'il abritait autant de statues que la ville de Rome tout entière ${ }^{4}$. Cette recherche effrénée d'œuvres d'art eut pour conséquence la perte irrémédiable des informations relatives aux contextes précis de découverte - dont la connaissance n'a d'ailleurs jamais compté parmi les préoccupations de ceux qui les ont exhumées. On en trouve une première confirmation éclatante dans les Antichità di Ercolano esposte (1757-1792), dont les volumes V et VI, consacrés aux sculptures en bronze ${ }^{5}$, ne mentionnent pratiquement aucun

Quotidien des dieux et des hommes. La vie religieuse dans les cités du Vésuve à l'époque romaine, Rome, 2009, p. XX-XXII.

3 On désigne ainsi les ouvriers spécialisés qui pratiquaient les fouilles à partir de tunnels creusés à la manière des mines.

4 J. J. Winckelmann, Geschichte der Kunst des Altertums, cité dans J. L. Jiménez Salvador (ed.), Bajo la cólera del Vesubio : testimonios de Pompeya y Herculano en la época de Carlos III, Valence, 2004, p. 159.

5 Volume V : publié en 1767, sur les bustes ; volume VI publié en 1771 sur les statues. Le texte intégral du volume VI est consultable et téléchargeable à l'adresse suivante : 


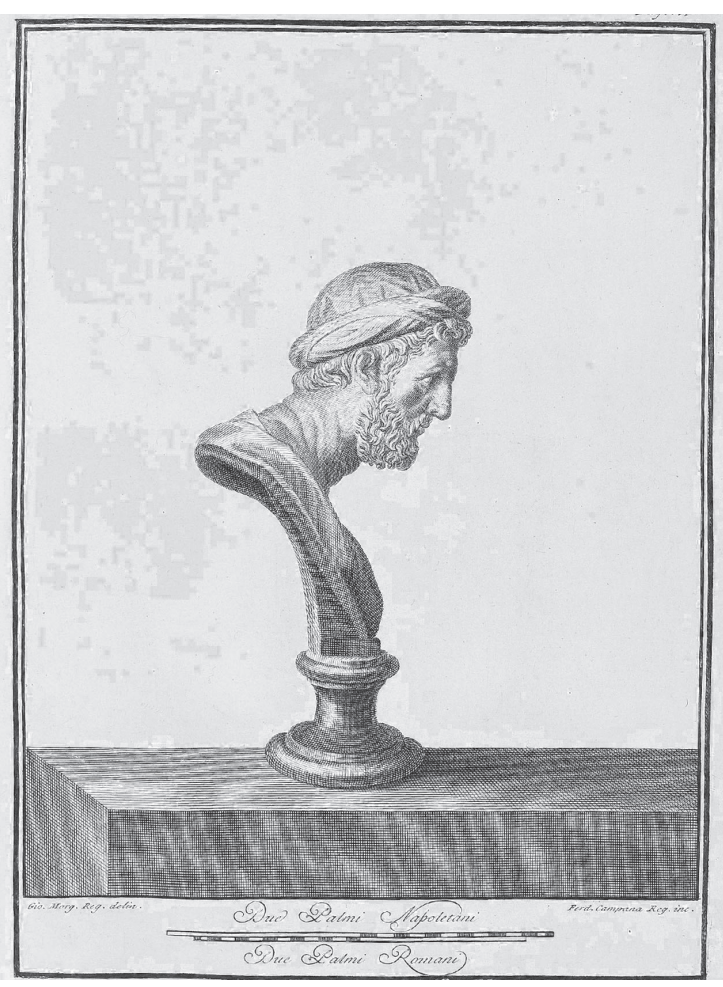

Fig. 1. Buste en bronze d'Archytas de Tarente, provenant de la villa des Papyri à Herculanum, d'après Antichità di Ercolano esposte. Bronzi di Ercolano, Volume I-Busti, Napoli, 1767, pl. XXX. lieu de découverte, alors que le plus grand soin est donné, dans les planches, aux détails de la représentation, qui fournit souvent les dimensions exactes de l'objet (fig. 1) et, dans le texte, à la description iconographique. Quant aux volumes consacrés aux marbres, ils n'ont jamais vu le jour, ce qui crée une lacune documentaire considérable, qui a toutefois été très partiellement compensée par la publication en 2006, sous la direction de M.Pagano et R. Prisciandaro, de rami inediti destinés aux Antichità di Ercolano ${ }^{6}$; par exemple, le très beau relief dionysiaque polychrome ${ }^{7}$ proviendrait de la Casa dei Cervi et aurait été retrouvé le 20 mars 1749 , tandis que le grand relief de la nymphe repoussant un satyre aurait été trouvé quelques semaines plus tôt, dans la Casa dell'Atrio Tuscanico $^{8}$. Paradoxalement, hormis pour les œuvres issues du théâtre ou de certains monuments publics du centre monumental

http://www.e-rara.ch/doi/10.3931/e-rara-937. Sur les statues, voir J.M. NoguerA Celdrán, “Ciclos estatuarios en Herculano, según Delle antichità di Ercolano. Tomo sesto: statue ", in J. L. Jiménez Salvador (ed.), Bajo la cólera del Vesubio, Valence, 2004, p. 153-201.

6 M. Pagano \& R. Prisciandaro, Studio sulle provenienze degli oggetti rinvenuti negli scavi borbonici del Regno di Napoli: una lettura integrata, coordinata e commentata della documentazione, Castellamare di Stabia, 2006.

7 Naples, MANN, inv. 6726 : H.-U. CAIN \& O. DräGER, “ Die sogenannten neuattischen Werkstätten ", in Das Wrack. Der antike Schiffsfund von Mahdia, Cologne, 1994, 810811, fig. 1 p. 810.

8 Naples, MANN, inv. 6724 : B. Hundsalz, Das dionysische Schmuckrelief, Diss. Köln 1986, cat. $\mathrm{n}^{\circ}$ K 91 p. 191 et suivantes. 
(Augusteum ou « basilique »), on connaît mieux les campagnes de restaurations ou les conditions d'exposition successives des statues du Museum Herculanense de Portici ou du Real Museo Borbonico de Naples que leur lieu de découverte. Ce relatif désintérêt est également confirmé par le caractère à la fois lacunaire et elliptique des descriptions présentes aussi bien dans les documents d'archive que dans les publications anciennes. Plusieurs dizaines de statues ou statuettes issues des explorations du XVIII siècle et actuellement conservées au Musée de Naples sont simplement inventoriées comme provenant d'Herculanum. Il est exceptionnel qu'une date, une maison ou un espace précis soient mentionnés.

À ces difficultés s'ajoutent, comme pour les autres témoignages d'Herculanum, la dispersion actuelle des objets, qui a conduit au démantèlement d'ensembles initialement cohérents : ainsi deux des pieds décorés d'une même table en marbre ont été insérés dans une table exposée aujourd'hui au Musée de Capodimonte, tandis que le troisième se trouve à Berlin? .

Le travail inverse, qui consiste non pas à connaître le lieu de découverte précis d'une pièce indiquée comme provenant d'Herculanum, mais à repérer dans les réserves une œuvre dont le lieu de découverte est connu par les journaux de fouille mais qui se trouve désignée par le seul personnage ou divinité représenté(e), n’est pas plus aisé. Si les dessins et gravures des pièces les plus remarquables ou les plus imposantes ne manquent pas, les illustrations d'œuvres considérées comme « mineures » sont rarissimes et leur description extrêmement sommaire ; seuls le croisement de sources et de données hétérogènes issues des publications anciennes, des inventaires successifs du Musée et des documents de fouilles (pour les trouvailles les plus récentes) permettent dans certains cas de sortir de l'impasse : les associations d'objets, la présence de motifs rares ou particulièrement remarquables et les dates de trouvaille sont d'une aide précieuse. Les notices relatives aux restaurations des œuvres en marbre aident également à fournir des termini ante quem pour certaines découvertes ${ }^{10}$.

Les clichés photographiques anciens doivent être exploités avec prudence: certains sont très précieux en ce qu'ils montrent les objets encore pris dans leur gangue de débris volcaniques ${ }^{11}$, tandis que d'autres, remontant aux années ayant immédiatement suivi les fouilles dirigées par A. Maiuri, loin de refléter fidèlement les circonstances et les lieux de découverte des objets ${ }^{12}$, illustrent

9 G. C. Ascione \& M. Pagano, The Antiquarium of Herculaneum, Milan, 2000, p. 51-54, fig. p. 54.

10 On trouve ces informations dans des documents d'archive mentionnant les frais occasionnés par la restauration des statues.

1 Cliché de la Surintendance de Naples : http://arachne.uni-koeln.de/item/marbilder bestand/931565

12 En outre, certains objets étaient au moment de l'éruption entreposés en dehors de leur lieu d'exposition originel. 
des regroupements d'objets à visée scénographique et touristique ${ }^{13}$. La statuette en bronze rehaussé d'or et d'argent représentant Dionysos nu, l'épaule gauche couverte de la pardalide ${ }^{14}$ (pl. V.1), trouvée dans “ l'atelier du plumbarius »(VI, 12), apparaît sur des clichés de fouilles de l'année 1961 à plusieurs emplacements différents avant d'être présentée aux visiteurs, après restauration, à l'angle externe de l'atelier (fig. 2). Si on ajoute à cela le fait que l'ouvrage sur la décoration et les œuvres d'art projeté par A. Maiuri en complément de la présentation générale du site, publiée en 1958, n'a jamais paru ${ }^{15}$, on mesure la fragilité de toute tentative de recontextualisation fine des sculptures, même pour la période des nuovi scavi, c'est-à-dire à partir de 1927. On l'aura compris, la sculpture des espaces domestiques demeure, avec le mobilier au sens large, l'un des types de décors les moins étudiés et les plus difficilement « recontextualisables » d'Herculanum.

\section{L'exemple des reliefs à décor de quadriges}

Pour mieux illustrer ces difficultés méthodologiques, je développerai tout d'abord un exemple relevant de la sculpture décorative domestique. Mes premières tentatives de recontextualisation des œuvres sculptées se sont portées sur les reliefs, car ils me paraissaient davantage susceptibles d'avoir été conservés à leur emplacement d'origine en raison de leur nature même, celle de pinakes ou typoi sculptés qui étaient fréquemment insérés dans la maçonnerie des murs des maisons elles-mêmes ${ }^{16}$. J'y étais encouragée par la découverte en 2009, dans une pièce ornée de peintures appartenant à un espace de représentation d'un édifice résidentiel de l'insula ${ }^{17}$, d'un relief mythologique figurant probablement l'épisode de la guérison des filles de Prœtus par le devin Melampus ${ }^{18}$. Il se

13 Voir l'exemple de la “ chambre de la brodeuse » analysé par N. Monteix, Les lieux de métier. Boutiques et ateliers d'Herculanum, Rome, 2010, p. 20-21, fig. 3.

14 Herculanum, dépôt archéologique, inv. E 2292 : T. Budetta \& M. Pagano, Ercolano: legni e piccoli bronzi. Testimonianze dell'arredo e delle suppellettili della casa romana. Roma, Castel S. Angelo, 23 marzo - 26 aprile 1988, Rome, 1988, cat. 30, p. 78-81, fig. p. 78, p. 110-111.

15 A. Maiuri, Ercolano. I nuovi scavi (1927-1958). II - La decorazione. Le opere d'arte : le volume est resté à l'état de projet.

16 Sur cette question, voir notamment L. E. BAumer, “Praeterea typos tibi mando. Klassische Weihreliefs im römischen Kontext », Hefte des archäologischen Seminars des Universität Bern 4 (2001), p. 85-94.

17 M.-P. Guidobaldi \& D. Esposito, Herculanum, Paris, 2012, p. 151-155.

18 Herculanum, dépôt archéologique, inv. 88091. Sur ce relief, voir M.-P. Guidobaldi \& P. G. Guzzo, “ Un rilievo neoattico da Ercolano », CronErcol 40 (2010), p. 251-260 et F. CAruso, “ Melampo e le figlie di Preto. Una lettura del nuovo rilievo da Ercolano », 

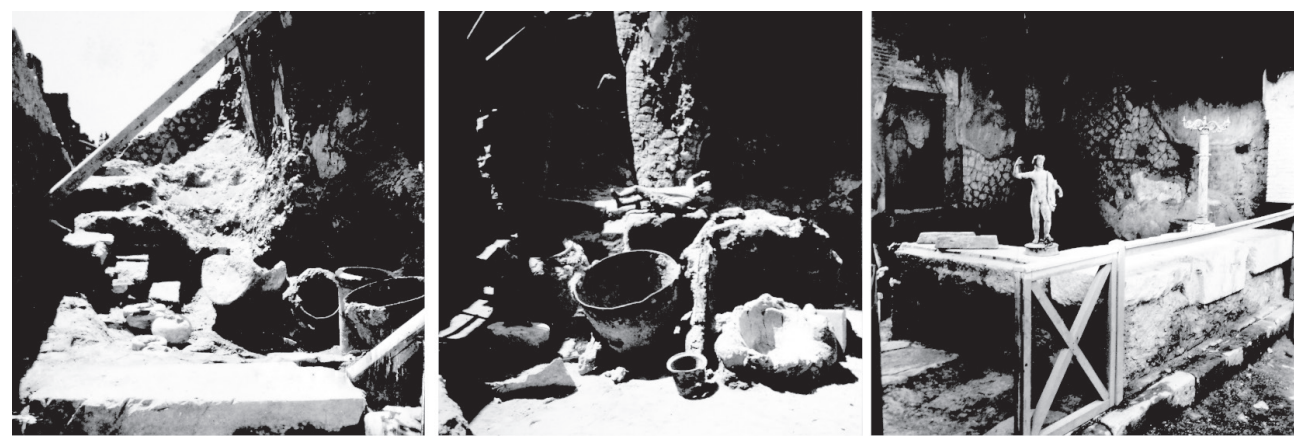

Fig. 2. L'atelier de plumbarius VI, 12 en cours de fouille et après restauration.

Clichés Parco archeologico di Pompei, de g. à dr. : D5922, juillet 1961; D5932, juillet 1961; A2704, novembre 1965.

trouvait encore in situ, encastré à une hauteur de deux mètres au-dessus du sol, au même niveau qu'un autre relief trouvé en 1997 sur la paroi sud de la même pièce $^{19}$ et figurant deux satyres et une nymphe buvant auprès d'une source ${ }^{20}$. Un tel exemple, conforté par celui du célèbre relief de Télèphe exposé dans la maison à laquelle il a donné son nom, invitait à étendre l'enquête à l'ensemble des attestations de ce genre de panneaux sculptés à Herculanum afin de préciser leur emplacement d'origine et par là-même étudier leur éventuelle complémentarité avec les peintures pariétales qui sont au cœur du programme ANR VESUVIA.

Deux séries de reliefs en marbre présentent l'intérêt de reproduire un même original, que certains ont considéré comme remontant au classicisme grec, mais qui relève plus probablement d'une production classicisante du début de l'Empire. Comme les reliefs dionysiaques précédemment évoqués, ces derniers appartiennent en effet au courant « néo-attique ».

La collection d'antiques du Duc de Loulé, à Lisbonne, comprenait deux reliefs en marbre pratiquement complets ${ }^{21}$ (fig. 3 a-b). Ils présentent une composition de

Rivista di Studi Pompeiani 22 (2011), p. 25-35 ; A. CiотоцA, “ Ancora sul rilievo neoattico di Ercolano: una diversa lettura », RStPomp 24 (2013), p. 33-40.

19 Herculanum, Dépôt archéologique, inv. 79613. Sur ce relief, voir S. Böнм, “ Labung an der Weinquelle. Ein dionysisches Marmorrelief in Herculaneum », JdI 123 (2008), p. 171-197.

20 Pour les conditions de fixation du relief au mur, voir http://www.archart.it/italiaercolano-gli-scavi-sorprendono-ancora-scoperto-un-rilievo-di-marmo-dartegreca.html.

21 Les reliefs sont aujourd'hui conservés à la fondation Bodmer, à Genève. Le matériau est désigné dans plusieurs publications comme du marbre pentélique. Ht. 0, 76 m ; L. 1, $45 \mathrm{~m}$. 


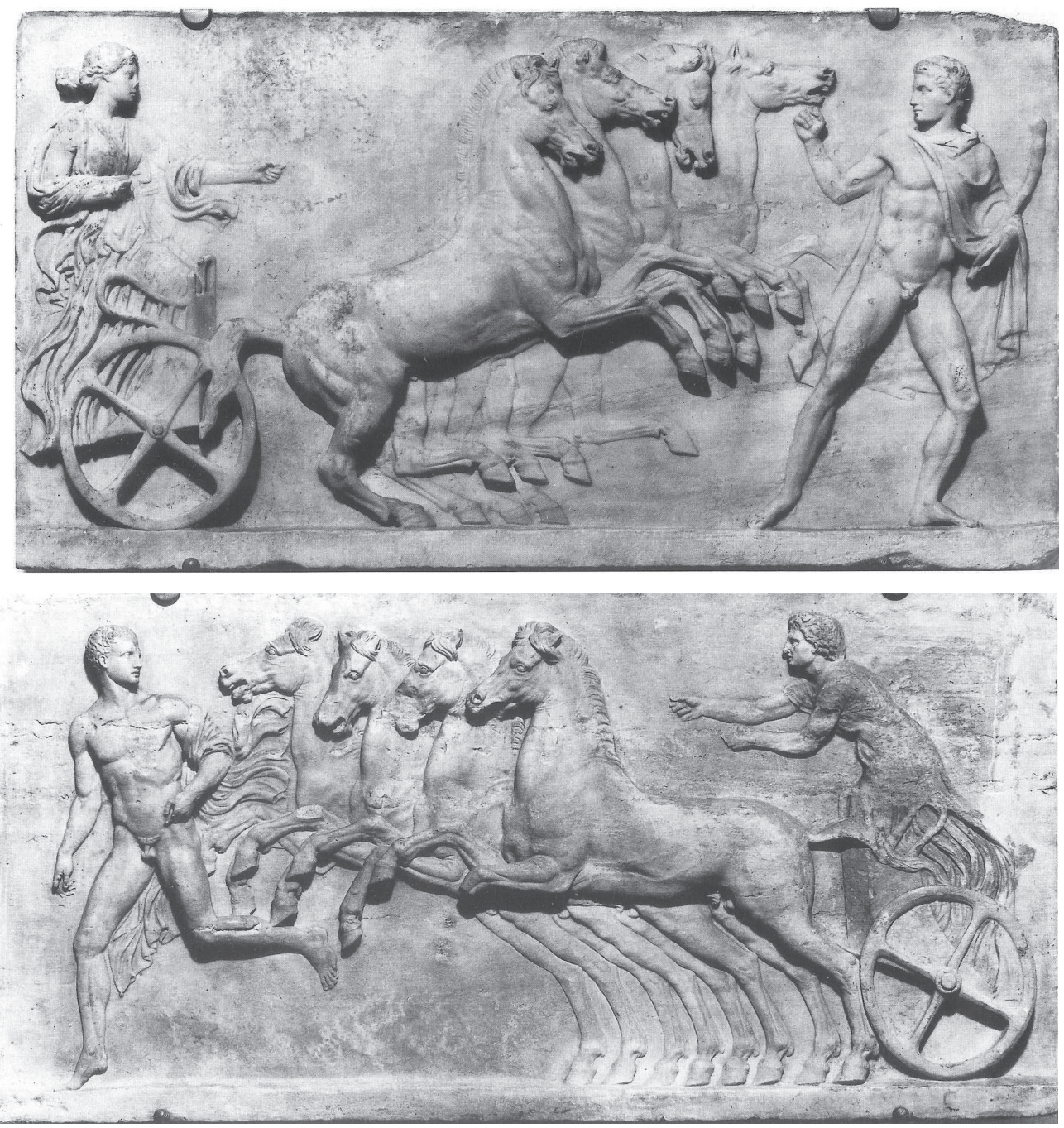

Fig. 3 a-b. Genève, fondation G. Bodmer - Reliefs de la collection Loulé, types A et B, provenant peut-être d'Herculanum. D’après M. C. Monaco, “ Il rilievo n.539 degli Uffizi e la serie neoattica Loulé », $B d A 95$ (1996), fig. 2 p. 86, fig. 3 p. 88. 
type héraldique, celle de deux quadriges de direction convergente dont chacun est conduit par une figure, l'une féminine, l'autre masculine, et précédé par un jeune homme portant la chlamyde qui court devant le char et le guide. On en connaît plusieurs paires, désignées comme "série Loulé22 » ou "série HerculanumÉphèse-Lisbonne ${ }^{23}$ » et exhumées dans des contextes fort différents, de Rome à Éphèse ${ }^{24}$. De nombreuses propositions d'identifications ont été formulées ; s'il existe un consensus sur leur nature de divinités astrales ou célestes, on y a vu d'abord Éôs et Phosphoros d'un côté, Hélios de l'autre ${ }^{25}$, avant d'identifier Phosphoros devant le char d'Héméra et Hespéros devant le char de Nyx.

De ces reliefs existaient des réductions en bronze et des moulages exposés au XIx ${ }^{e}$ siècle au Musée de sculpture comparée du Trocadéro à Paris ${ }^{26}$; ils ont suscité un débat assez vif auprès des spécialistes à la fin du $\mathrm{xIX}^{\mathrm{e}}$ siècle. Comme certains, dont Paul Wolters ${ }^{27}$, doutaient de leur authenticité, la question de leur provenance est devenue cruciale. Une première tradition rapportait qu'ils avaient été acquis à Rome auprès d'un antiquaire qui les disait issus de Pompéi ${ }^{28}$. Mais d'après un autre témoignage recueilli par F. G. Welcker, ils proviendraient en réalité d'Herculanum ${ }^{29}$; quoiqu'aucune autre source ne vienne la corroborer, cette provenance est indiquée dans la plupart des publications plus récentes qui ont évoqué ces reliefs. Le Duc de Marialva ayant été ambassadeur en 1775 à la cour de Naples, M. Boutroue en faisait remonter l'acquisition ou le don à cette période ${ }^{30}$. Si un don personnel du roi de Naples paraît peu probable, comme le note A. Maiuri ${ }^{31}$, en raison de l'importance que le roi attachait aux premières découvertes d'Herculanum, il reste pour cette période la possibilité

22 M. C. Monaco, “ Il rilievo n.539 degli Uffizi e la serie neoattica Loulé », BdA 95 (1996), p. 85-104.

23 Ch. Picard, “Sur les reliefs dits du Duc de Loulé à Lisbonne », Annuario della Scuola Archeologica di Atene e delle Missioni Italiane in Oriente 24-26 (1946-48), 1950, p. 214.

24 F. Slavazzi, "Il ciclo dei rilievi nella “Kaisersaal» del Ginnasio di Vedio a Efeso », in Iconografia 2005. Atti del Convegno Internazionale (Venezia, 26-28 gennaio 2005), Rome, 2005, p. 235-243.

25 Résumé des propositions d'identification dans Picard, “Sur les reliefs », p. 218-220.

26 T. Homolle, "Deux bas-reliefs néo-attiques du Musée de Lisbonne ", BCH 16-1 (1892), n. 3 p. 325, p. 327. Cf. Musée de sculpture comparée, 1883, p. 52, n 303-305.

27 Friederichs-Wolters, Gipsabgüsse, no 1838.

28 Homolle, “ Bas-reliefs », p. 327.

29 F. G. Welcker, Das Akademische Museum zu Bonn, Bonn, 1841, p. 122, n 389-390. C'est ce qu'indiquaient également les cartels du Musée de sculpture comparée du Trocadéro.

30 Boutroue, in Gazette des Beaux-Arts 1882, I, p. 556 ; Homolle, « Bas-reliefs », p. 327.

31 A. Maiuri, “ Rilievi con quadrighe da Ercolano », Annali della Scuola Archeologica di Atene e delle Missioni Italiane in Oriente 24-26 (1946-48), 1950, p. 221. 
qu'elles proviennent de la fouille du théâtre, qui comme on le sait, fut fouillé clandestinement par le Prince d'Elbœuf et dont les découvertes furent dispersées dans des collections publiques et privées. La concentration de telles œuvres néoattiques dans les théâtres de l'Occident romain - en Gaule mais aussi en Espagne pourrait donner quelque crédit à cette hypothèse, mais elle reste à l'heure actuelle totalement invérifiable.

Néanmoins la découverte en contexte de fouille, durant les années 1933-1934, de fragments de deux autres plaques ${ }^{32}$ présentant une iconographie pratiquement identique $^{33}$ ajoute un élément intéressant au débat. Elles relèvent en effet de campagnes bien mieux documentées que celles des époques précédentes ${ }^{34}$. Une dizaine de fragments appartenant à deux plaques symétriques a été publiée en 1950 par A. Maiuri, qui en donne une étude contextuelle détaillée et recense une partie des répliques connues ailleurs dans le monde romain ${ }^{35}$ (fig. 4 a-b). Il en précise les lieux de découverte successifs, qui illustrent parfaitement la dispersion des œuvres et de leurs fragments sous l'effet de la coulée pyroclastique (fig. 5). Il s'agit d'un véritable cas d'école, qui invite à la plus grande prudence dès lors que l'on se donne pour objectif de restituer l'emplacement des sculptures.

Les fragments sont mentionnés dans trois types de documents différents : les carnets du Giornale degli scavi di Ercolano, les libri dei Trovamenti et enfin un “ Inventaire des marbres » de deux pages conservé à l'Archivio Storico de Naples ${ }^{36}$, donnent pour certains d'entre eux, à côté d'une description très sommaire des fragments, les coordonnées spatiales de leur découverte, indiquées en plan par rapport aux parois et en hauteur par la distance au-dessus du niveau de sol ; les données en sont compilées dans le tableau fig. 6. La liste complémentaire à l'inventaire fait l'objet d'un traitement complètement séparé de la description des maisons elles-mêmes ; elle est pourtant écrite de la même main et est donc vraisemblablement contemporaine. On pourrait par conséquent émettre l'hypothèse selon laquelle cet inventaire constitue un document synthétique préparatoire à la rédaction de l'article destiné au volume des Annales de l'École italienne d'Athènes et à la restauration des reliefs, ce qui justifierait que soient regroupées les informations les concernant.

32 Maiuri, "Rilievi con quadrighe », pl. XXIII-XXIV; Monaco, " Il rilievo n.539 degli Uffizi », fig. 7 p. 90 , fig. 8 p. 91.

33 La différence principale réside dans l'ajout, sur le relief du type A, d'un pilier surmonté d'une statuette d'Apollon archaïsant à gauche de la composition.

34 Après avoir été un temps exposés dans la maison du relief de Télèphe, ils sont actuellement présentés dans la Casa del Bel Cortile.

35 Maiuri, “ Rilievi con quadrighe », p. 221-228.

36 Naples, Archivio Storico, inv. 145. 

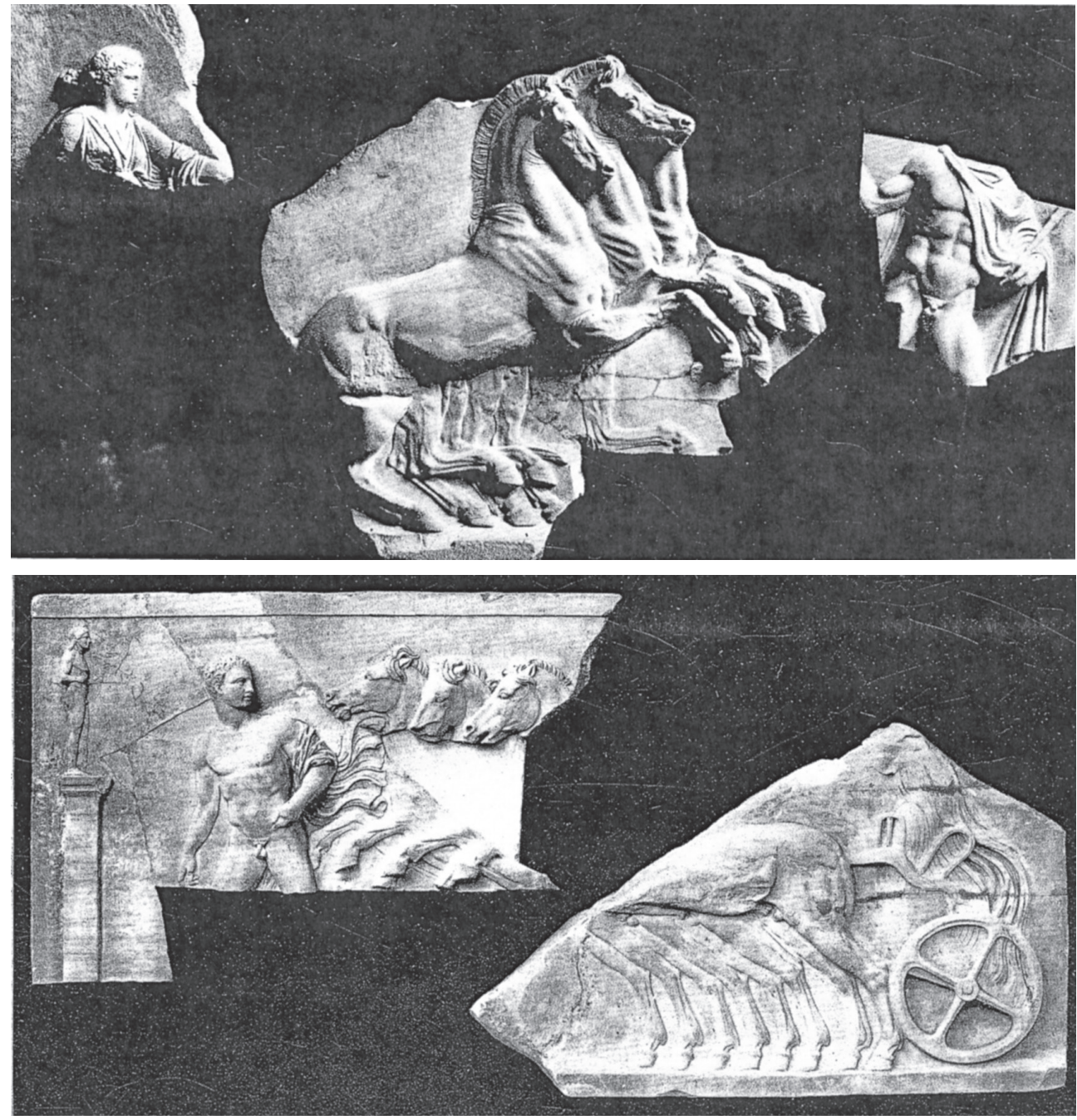

Fig. 4 a-b. Reliefs A et B de la série néo-attique Loulé, provenant d’Herculanum.

D'après A. Maiuri, "Rilievi con quadrighe da Ercolano ", Annali della Scuola Archeologica di Atene e delle Missioni Italiane in Oriente 24-26 (1946-48), 1950, pl. XXIII-XXIV. 


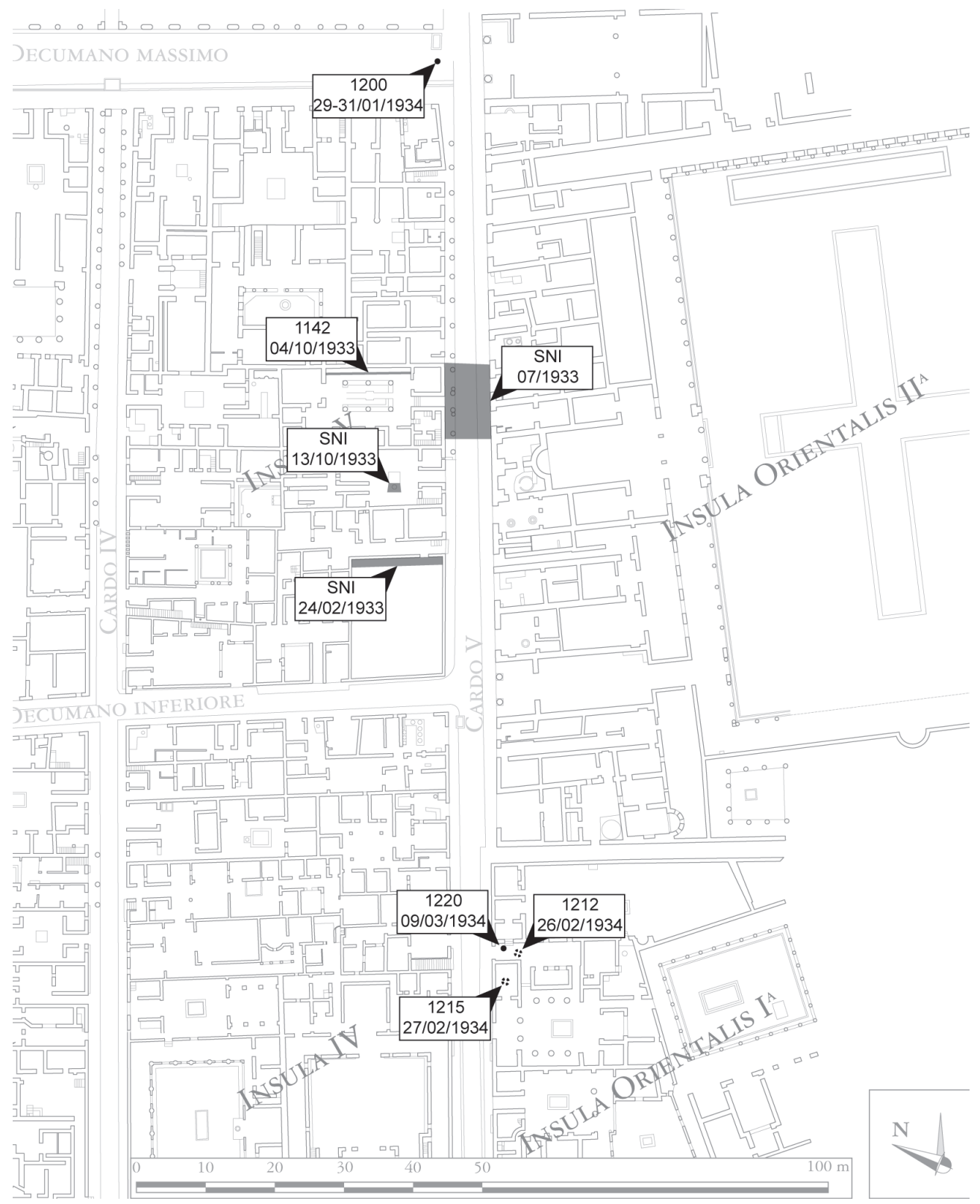

Fig. 5. Plan d'Herculanum, avec l'indication des lieux et dates de provenance des fragments de plaque de la série Loulé. Infographie N. Monteix. 


\begin{tabular}{|c|c|c|c|}
\hline $\begin{array}{l}\text { Date de } \\
\text { découverte }\end{array}$ & $\begin{array}{l}\text { Description des fragments } \\
\text { (d'après le Giornale degli } \\
\text { scavi di Ercolano, } \\
\text { sauf indication contraire) }\end{array}$ & $\begin{array}{l}\text { Lieu de } \\
\text { découverte }\end{array}$ & $\begin{array}{l}\text { Numéro } \\
\text { d'inventaire }\end{array}$ \\
\hline 24/02/1933 & $\begin{array}{l}\text { Fragment figurant un quagrige incomplet } \\
\text { ("Sul muro nord è stata raccolta una } \\
\text { bella scultura in marmo, che rappresenta } \\
\text { una quadriga, però non è completa. È di } \\
\text { finissima scultura. Manca tutta la parte } \\
\text { posteriore, e per quante minute ricerche } \\
\text { sono state fatte, non abbiamo nulla } \\
\text { rintracciato") }\end{array}$ & $\begin{array}{l}\text { Jardin de la maison } \\
\mathrm{n}^{\circ} 1, \text { cardo } \mathrm{V} \text {, côté } \\
\text { ouest }(=\text { Casa con } \\
\text { giardino } \mathrm{V}, 33)\end{array}$ & SNI \\
\hline $24 / 06 / 1933$ & $\begin{array}{l}\text { Fragment figurant une statue d'Apollon } \\
\text { sur un pilier } \\
\text { («Bassorilievo con la figura di Apollo } \\
\text { greco. Con la mano sinistra stringe l'arco } \\
\text { che serviva per la freccia e la destra la } \\
\text { tiene chiusa presso l'addome. Libr. Trov. } \\
\mathrm{N}^{\circ} 1107 \text {. I capelli sono lunghi e parte di } \\
\text { essi scendono sul petto e la rimanenza } \\
\text { lungo il dorso. Ė incompleto ») }\end{array}$ & $\begin{array}{l}\text { Pièce } 3 \text { de la } \\
\text { maison n }{ }^{\circ} 4, \text { cardo } \\
\text { V, côté ouest, sur le } \\
\text { sol (= Casa dell'atrio } \\
\text { corinzio) }\end{array}$ & $\begin{array}{l}\text { Inv. } \mathrm{n}^{\circ} 1107 \\
(=76384) \\
(\text { relief A) }\end{array}$ \\
\hline $4 / 10 / 1933$ & $\begin{array}{l}\text { Fragment figurant trois têtes de chevaux } \\
\text { portant des traces de polychromie rouge } \\
\text { ("Parte superiore di una quadriga. Vi sono } \\
\text { a rilievo tre teste di cavallo. Il pezzo è largo } \\
\text { m. } 0.56 \text { ed alto m. } 0.24 \text { e dello spessore di } \\
\text { m. } 0.03 \text {. Al cavallo centrale manca il muso. } \\
\text { Il tutto è di una discreta fattura") }\end{array}$ & $\begin{array}{l}\text { Maison } \mathrm{n}^{\circ} 4, \text { cardo } \\
\text { V, côté ouest, } \\
\text { ambulacre côté } \\
\text { nord, à } 1,75 \mathrm{~m} \text { au- } \\
\text { dessus du niveau } \\
\text { de sol et à } 2,70 \mathrm{~m} \\
\text { de l'entrée de la } \\
\text { pièce } 3\end{array}$ & $\begin{array}{l}\text { Inv. } n^{\circ} 1142 \\
(=76419) \\
(\text { relief A) }\end{array}$ \\
\hline 13/10/1933 & $\begin{array}{l}\text { Fragment figurant des jambes de chevaux } \\
\text { appartenant au quadrige trouvé le } \\
\text { 24/02/1933 (cf. ci-dessus) } \\
\text { ("frammenti di marmi e precisamente } \\
\text { delle zampe di cavalli che appartengono } \\
\text { alla quadriga che trovasi nel giardino della } \\
\text { casa n. } 1 \text { sullo stesso cardine e lato") }\end{array}$ & $\begin{array}{l}\text { Maison } n^{\circ} 3, \text { cardo } \\
\text { V, côté ouest, insula } \\
\text { meridionale, près } \\
\text { du puteal de terre } \\
\text { cuite qui se trouve } \\
\text { sur le côté sud de } \\
\text { l'impluvium }\end{array}$ & SNI (relief A) \\
\hline
\end{tabular}

Fig. 6. Tableau synthétique : lieu, date et circonstances de découverte des fragments de reliefs*.

* Dans ce tableau ne figurent que les fragments dont le lieu de découverte est mentionné dans le GSE. D'autres fragments appartenant aux deux reliefs sont présents dans les inventaires, mais sans indication de provenance (inv. 2576 et 2579 ). 


\begin{tabular}{|c|c|c|c|}
\hline 31/01/1934 & $\begin{array}{l}\text { Fragment d'un quadrige en marbre blanc } \\
\text { (Naples, feuillet d'inventaire des marbres } \\
\text { associés aux quadriges, inv. } 145 / \mathrm{f}^{\circ} 5: \\
\text { "parte di una quadriga di marmo bianco ») }\end{array}$ & $\begin{array}{l}\text { "Trivio del } \\
\text { decumano } \\
\text { massimo », à } 2,70 \mathrm{~m} \\
\text { de la fontaine et à } \\
2,25 \mathrm{~m} \text { du cardo } \mathrm{V}, \\
\text { sur le sol }\end{array}$ & $\begin{array}{l}\text { Inv. } 1200 \\
(=76477)\end{array}$ \\
\hline 26/02/1934 & $\begin{array}{l}\text { Marbre blanc figurant la tête d'un } \\
\text { personnage masculin de profil. } \\
\text { ("una bellissima testa di Ercole (?), } \\
\text { policromato rosso. È vista di profilo, tiene } \\
\text { il naso profilato, i capelli arruffati e il collo } \\
\text { ben tornito") }\end{array}$ & $\begin{array}{l}\text { Maison } \mathrm{n}^{\circ} 2 \text {, à } \\
\text { l'extrémité sud } \\
\text { du cardo } \mathrm{V} \text {, côté } \\
\text { est (=Casa del } \\
\text { Rilievo di Telefo), } \\
\text { "in un cunicolo } \\
\text { borbonico » }\end{array}$ & $\begin{array}{l}\text { Inv. } 1212 \\
(=76490) \\
\text { (relief A) }\end{array}$ \\
\hline $27 / 02 / 1934$ & $\begin{array}{l}\text { Fragment figurant le buste d'une figure } \\
\text { féminine de profil à droite } \\
\text { ("Parte di lastra con un bellissimo alto } \\
\text { rilievo. Misura m. } 0.255 \text { di larghezza, } \\
\text { m. } 0.03 \text { di spessore e m. } 0.24 \text { di altezza. } \\
\text { Rappresenta parte di una donna, mancante } \\
\text { dell'avambraccio e mano destra, come } \\
\text { pure la mano la sinistra. Tiene avvolta al } \\
\text { braccio sinistro una tunica. Il collo è ben } \\
\text { tornito e il naso è profilato. I capelli sono } \\
\text { lunghi e annodati all'estremità. Il petto } \\
\text { è sporgente. Le braccia sono coperte a } \\
\text { metà, è di fattura greca. Rappresenta una } \\
\text { Ninfa?"). }\end{array}$ & $\begin{array}{l}\text { Maison n } 2 \text { 2, à } \\
\text { l'extrémité sud du } \\
\text { cardo } \mathrm{V} \text {, côté est } \\
\text { (=Casa del Rilievo } \\
\text { di Telefo), pièce } \\
\text { non numérotée, à } \\
0,27 \mathrm{~m} \text { au-dessus } \\
\text { du sol }\end{array}$ & $\begin{array}{l}\text { Inv. } \mathrm{n}^{\circ} 1215 \\
(=76493) \\
(\text { relief B) }\end{array}$ \\
\hline 09/03/1934 & $\begin{array}{l}\text { Fragment figurant le torse d'un homme nu } \\
\text { acéphale } \\
\text { ("a rilievo un uomo nudo mancante della } \\
\text { testa e degli arti inferiori. Il braccio destro } \\
\text { è disteso e la mano tiene le dita piegate. } \\
\text { Al sinistro è avvolto un velo e la mano } \\
\text { poggia sul fianco dello stesso lato. È visto } \\
\text { di profilo. L'uomo, Ercole(?), è raggiunto } \\
\text { dalle zampe di quattro cavalli attaccati } \\
\text { ad una quadriga. Dei quattro animali si } \\
\text { osservano solo le zampe e della quadriga } \\
\text { nessuna traccia") }\end{array}$ & $\begin{array}{l}\text { Maison n }{ }^{\circ} 2, \text { à } \\
\text { l'extrémité sud du } \\
\text { cardo V, côté est } \\
\text { (= Casa del Rilievo } \\
\text { di Telefo), dans le } \\
\text { vestibule, à } 0,7 \mathrm{~m} \\
\text { de la paroi nord et } \\
\text { à } 0,25 \mathrm{~m} \text { au-dessus } \\
\text { du niveau du sol }\end{array}$ & $\begin{array}{l}\text { Inv. } 1220 \\
(=76498) \\
\text { (relief A) }\end{array}$ \\
\hline
\end{tabular}

Des fragments sont découverts dès le début de l'année 1933 dans la maison avec jardin $(\mathrm{V}, 33)$, mais ne figurent pas dans les inventaires. Quelques mois plus tard, de l'ensemble de fragments appartenant au « relief A » (pl. V.2), présentant le quadrige progressant vers la gauche, les deux premiers (inv. 1107 et 1142) ont été exhumés à l'intérieur de la Casa dell'Atrio Corinzio, tandis qu'un troisième 
ensemble (sans numéro d'inventaire) provient de la Casa del sacello di legno, et que la partie arrière du quadrige (inv. 1200) est issue de la zone du carrefour formé par le croisement du decumanus maximus et du cardo $\mathrm{V}$; enfin, un dernier fragment (inv. 1220) est trouvé l'année suivante dans l'atrium de la Casa del rilievo di Telefo (pl. VI.1).

Le « relief B » est quant à lui attesté par trois fragments : le premier (inv. 1200) a été récupéré en janvier 1934 à l'extrémité nord du cardo V, près de la porte ; le deuxième (inv. 1215- pl. VI.2) a été exhumé dans l'une des pièces de la Casa del rilievo di Telefo. Enfin, un dernier fragment appartenant sans aucun doute à ce relief (le format, le style et le sens de marche de la figure masculine représentée l'attestent) n'est pas recensé dans cette liste : découvert à une date bien antérieure et à un emplacement inconnu, il avait fait l'objet d'une restauration ${ }^{37}$. A. Maiuri, ayant repéré son appartenance à l'ensemble qu'il avait mis au jour, l'a intégré à juste titre à sa publication de 1950 et à la restauration de la plaque (fig. 4a); de fait, en 2014, c'est un relief entièrement moderne, présentant en son centre une lacune correspondant aux contours du fragment antique, que j'ai pu étudier dans les réserves du Musée de Naples (pl. VI.3).

Il est particulièrement instructif que la découverte de ces deux plaques lacunaires s'étale sur près de dix mois et que les fragments qui les composent soient issus de six secteurs différents - pas moins de quatre maisons distinctes, outre la rue elle-même. Comme le note A. Maiuri, entre les points les plus extrêmes des lieux de découverte la distance n'est pas inférieure à 120 mètres ${ }^{38}$ (fig. 5). Les cotes indiquant la hauteur des fragments par rapport au sol sont également riches d'enseignements, puisqu'elles varient de 0,25 à $1,75 \mathrm{~m}$.

Cet exemple analysé en détail permet de mesurer la complexe stratigraphie documentaire dont doit procéder toute étude de sculpture provenant d'Herculanum. A. Maiuri note avec justesse que les fragments ont en commun d'avoir été trouvés pour la plupart le long du cardo $\mathrm{V}^{39}$. La dispersion des fragments reflète et suit la trajectoire des coulées boueuses qui se sont engouffrées dans les maisons. Il est hautement probable que les deux reliefs aient formé des pendants dans un même espace qu'il n'est pas possible de qualifier davantage aujourd'hui, mais qui se situait au-delà du decumanus maximus - peut-être le vestibule supérieur de la « palestre ». Dans ces conditions, il serait bien imprudent de proposer un lieu et un contexte d'exposition pour les reliefs à décors de quadrige, pourtant bien documentés.

37 Naples, MANN, inv. 6680. Pour un cliché du relief restauré avant “ prélèvement » du fragment antique, voir MaIUR , “ Rilievi con quadrighe », fig. 3.

38 Maiuri, “ Rilievi con quadrighe », p. 222.

39 Ibid., p. 221. 
Les reliefs du type Loulé ne constituent pas un cas isolé ; en témoignent aussi les objets arrachés aux étages supérieurs des bâtiments et découverts bien au-dessus du niveau de circulation antique, ou les fragments d'une même œuvre trouvés à plusieurs mètres de distance. Quelques exemples particulièrement spectaculaires suffisent à mesurer l'ampleur des bouleversements et des déplacements : si un grand candélabre de marbre a été découvert quatre mètres au-dessus du niveau du sol ${ }^{40}$ dans la grande palestre, les éléments de l'étonnante fontaine en bronze figurant l'Hydre de Lerne qui ornait à l'origine le bassin cruciforme de la “ palestre », se trouvaient à près de trois mètres du socle initial au moment de leur découverte $^{41}$. De même, le plafond en bois de la “ salle des marbres » de la Casa del rilievo di Telefo a été arraché, renversé et précipité sur la plage d'Herculanum en contrebas ${ }^{42}$. On l'aura compris, à Herculanum, les lieux de découverte, même lorsqu'ils sont connus, ne peuvent en aucun cas être confondus avec les lieux d'exposition originels.

\section{Retour sur la « Palestre »}

Aussi est-on particulièrement étonné de voir certaines propositions d'identification de bâtiments fondées exclusivement sur la présence d'objets sculptés, parfois de dimensions très modestes, qui ont pu de la même façon être déplacés sur de longues distances. Je ne prendrai qu'un exemple relatif au même secteur de la cité, puisqu'il s'agit de la cosiddetta “Palestre ». Reprenant l'ensemble des données planimétriques, architecturales, épigraphiques et décoratives disponibles, Valentino Gasparini a proposé en 2010 d'identifier le complexe monumental comme un lieu de culte dédié au culte conjoint de la Mater Deum et d'Isis ${ }^{43}$. Or si la première divinité titulaire est formellement attestée par l'inscription dédicatoire mentionnant la restauration effectuée sous l'égide de l'empereur Vespasien, l'identification du culte isiaque repose sur le constat d'une concentration de témoignages aux abords immédiats des principaux accès au monument ou à l'intérieur de certaines salles ${ }^{44}$ (fig. 7). Les premiers sont de petits objets provenant de boutiques donnant sur le cardo $\mathrm{V}$, qui a naturellement

40 Herculanum, dépôt archéologique, inv. 78341 : Ascione \& Pagano, The Antiquarium of Herculaneum, $\mathrm{n}^{\circ} 18$ p. 86 (fig.).

41 Herculanum, dépôt archéologique, inv. 79242: A. Maruri, “ Fontana monumentale in bronzo nei nuovi scavi di Ercolano », Bollettino d'Arte III (1954), p. 193-199.

42 D. Camardo \& N. Notomista, “The roof and suspended ceiling of the marble room in the House of the Telephus relief at Herculaneum ", JRA 28 (2015), p. 39-70.

43 V. Gasparini, “La 'Palaestra' d'Herculanum: un sanctuaire d'Isis et de la Mater Deum », Pallas 84 (2010), p. 229-264.

44 Gasparini, “Palaestra », planche V p. 257. 


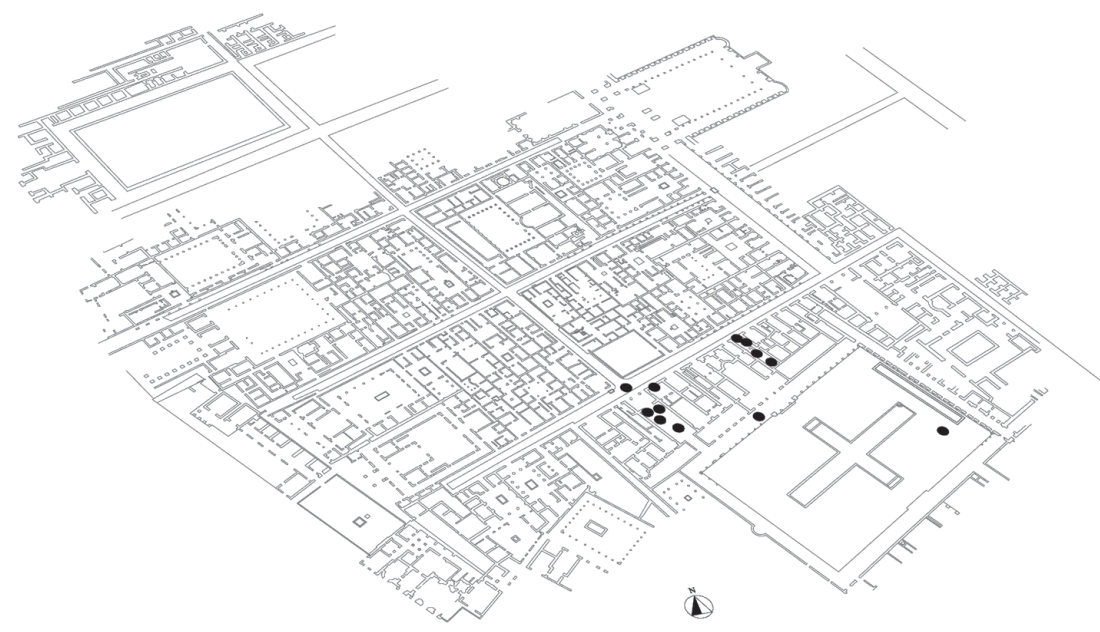

Fig. 7. Emplacement des lieux de découverte du « matériel isiaque » de la “ palestre » d'Herculanum. D’après V. Gasparini, “ La 'Palaestra' d'Herculanum : un sanctuaire d'Isis et de la Mater Deum », Pallas 84 (2010), pl. V-1 p. 257.

constitué, comme on l'a vu, l'un des principaux axes de circulation nord-sud de la coulée de débris : des pendentifs, un sistre en bois et une statuette d'Isis en terre cuite $^{45}$ qui, en dépit de sa qualité d'exécution, ne saurait valider la présence d'un sanctuaire à proximité. Les autres objets, considérés comme plus significatifs par V. Gasparini, sont en réalité des statuettes en bronze figurant Isis Fortuna, Bès ${ }^{46}$ et Harpocrate ${ }^{47}$, dont la plus grande n'excède pas $30 \mathrm{~cm}$ en hauteur, et une statue d'Atum en basalte d'une hauteur de $90 \mathrm{~cm}^{48}$, mais qui a été retrouvée brisée en trois fragments et, surtout, à deux mètres au-dessus du niveau de circulation

45 Herculanum, dépôt archéologique, inv. 1446 ; Gasparinı, “ Palaestra », p. 233, n. 41 (avec bibl. antérieure), pl. II-1 p. 254. Ht. 29, 5 cm.

46 Herculanum, dépôt archéologique, inv. E 1429 : Budetta \& Pagano, Legni e piccoli bronzi, cat. 22 p. 62-63, fig. p. 62 ; Gasparini, “ Palaestra », pl. III-3 p. 255.

47 Herculanum, dépôt archéologique, inv. 1420 ; Gasparinı, " Palaestra », p. 233, n. 44 (avec bibl. antérieure), pl. III-2 p. 255.

48 Herculanum, dépôt archéologique, inv. 2168 ; G. Bоттı, "Statuetta del dio Atum da Ercolano », $B d A 48$ (1963), p. 1-4. Gasparini, “Palaestra », n. 52 p. 234 (avec bibl. antérieure), pl. IV-4 p. 256. 
de la palestre. On peut difficilement éluder ce point et considérer que l'œuvre a été trouvée in situ, ou à proximité immédiate de son lieu d'installation originel. Comme l'avait suggéré A. Maiuri, suivi par G. Botti ${ }^{49}$ puis V. Catalano ${ }^{50}$ notamment, il est hautement probable que la statue a « circulé » sur une distance impossible à préciser aujourd'hui depuis un édifice situé en amont. On peut en dire autant de la petite base en bronze en forme de naos retrouvée dans le même secteur ${ }^{51}$. Par conséquent, outre le fait que la concentration est toute relative, puisqu'il s'agit d'une douzaine d'objets au total, on note que la plupart d'entre eux relève d'usages et de fonctions assez hétérogènes (bijoux ou amulettes, statuettes proches par leur typologie de celles issues de laraires, œuvres de plus grand format) et qu'une partie a été découverte non seulement en dehors du complexe monumental, mais encore à une hauteur et un emplacement qui interdisent de leur assigner la "palestre » comme lieu d'exposition au moment de l'éruption du Vésuve - et donc de déduire de leur présence la destination de l'édifice.

\section{Conclusion}

Par sa richesse et sa variété, le corpus des objets sculptés d'Herculanum paraît en première lecture constituer l'un des rares ensembles suffisamment contextualisés pour autoriser une réflexion approfondie sur le programme décoratif des édifices publics et privés d'une cité romaine au I ${ }^{\text {er }}$ siècle ap. J.-C. et sur la façon dont les objets figurés sont susceptibles d'éclairer les usages ou les fonctions des édifices dont ils sont issus. Néanmoins, du fait des effets conjugués de la coulée pyroclastique, qui a arraché la plupart des objets de taille modeste à leur base et à leur emplacement d'origine, des circonstances de dégagement du site durant les périodes les plus anciennes de la redécouverte et de l'absence detémoignages précis relatifs à l'emplacement des trouvailles (y compris pour des périodes relativement récentes), seule une infime partie des sculptures, situées dans des espaces préservés des coulées de boue, peut être considérée comme ayant été découverte in situ. Nous en sommes donc le plus souvent réduits aux conjectures et à une appréhension isolée des œuvres, selon une situation assez semblable en définitive à celle que connaît le spécialiste de statuaire antique pour la plupart des sites et des contextes. Si la tentation est grande d'exploiter la richesse documentaire des cités vésuviennes en ce sens, l'étude de certains ensembles mieux documentés

49 Вотті, “Statuetta del dio Atum », p. 4 (cite une lettre d'A. Maiuri).

50 V. Catalano, Abitanti e culti di Ercolano. Nuova edizione con gli indices, a cura di L. Garcia, G. Panzera, Rome, 2002, p. 156.

51 Naples, MANN, inv. 1107 ; GASParini, “ Palaestra », p. 233 n. 43 (avec bibl. antérieure), pl. IV-1 p. 256. 
invite en réalité à la plus grande prudence : si la qualité de l'information disponible peut parfois sembler décevante au regard des contextes exceptionnellement préservés de ces cités, la réflexion méthodologique qu'autorisent ces études de cas ponctuelles est extrêmement précieuse. La complexité des situations attestées - comme celle du Dionysos en bronze de l'atelier du plumbarius, retrouvé détaché de son socle et qui se trouvait probablement dans cet endroit pour réparation et non en tant qu'ornement de l'atelier - suggère de ne pas céder aux facilités interprétatives qu'induit une information tronquée.

\section{Emmanuelle Rosso Caponio}

Maître de conférences HDR en histoire de l'art et archéologie du monde romain Université Paris Sorbonne - EA 4081 - IUF Institut d'Art et d'Archéologie 3, rue Michelet - 75006 Paris emmanuelle.rosso@paris-sorbonne.fr 
Anabases 27 (2018), pl. I-XII.

\section{Restituer Herculanum II planches}

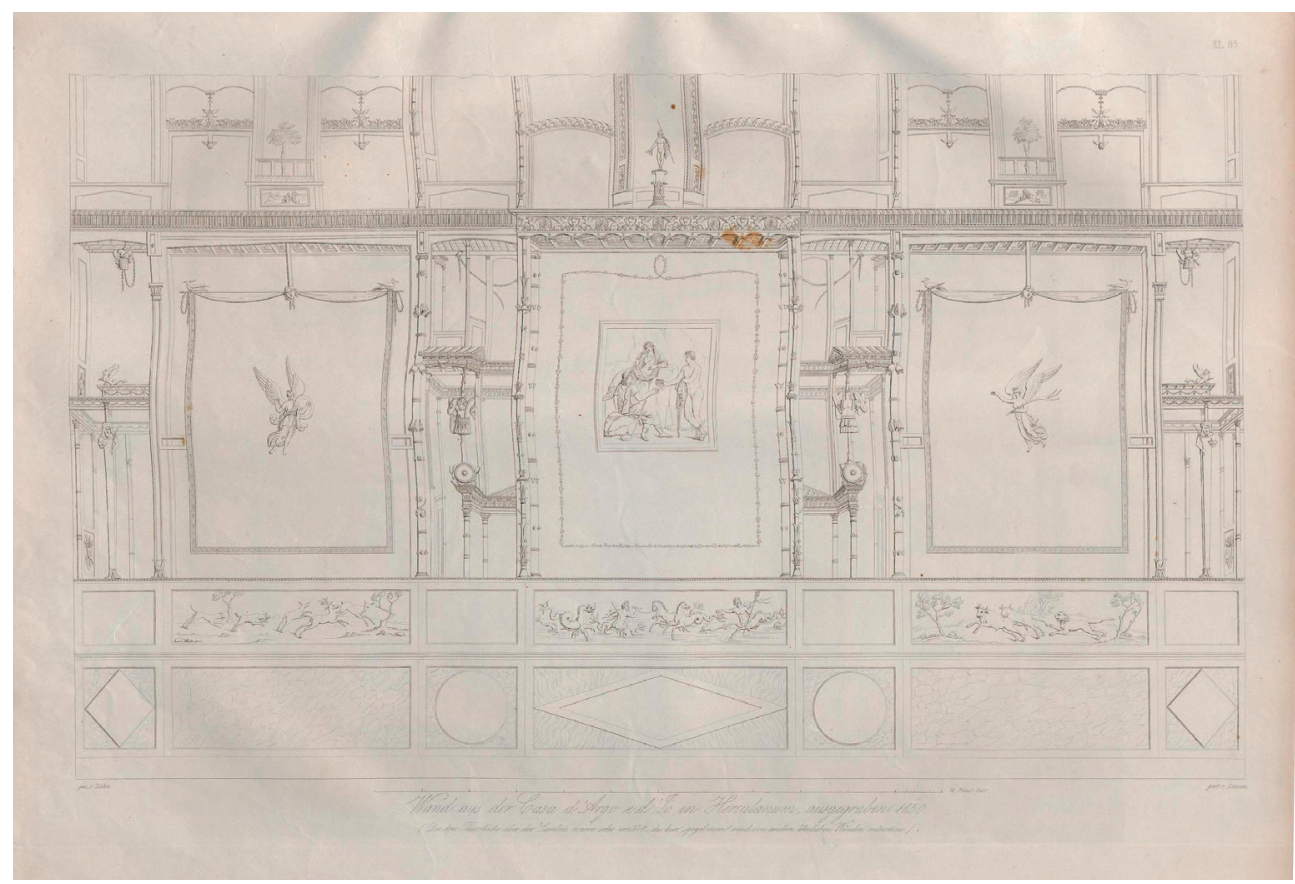

1. Paroi du triclinium, d'après W. Zahn, Les plus beaux ornements, tableau 83. 


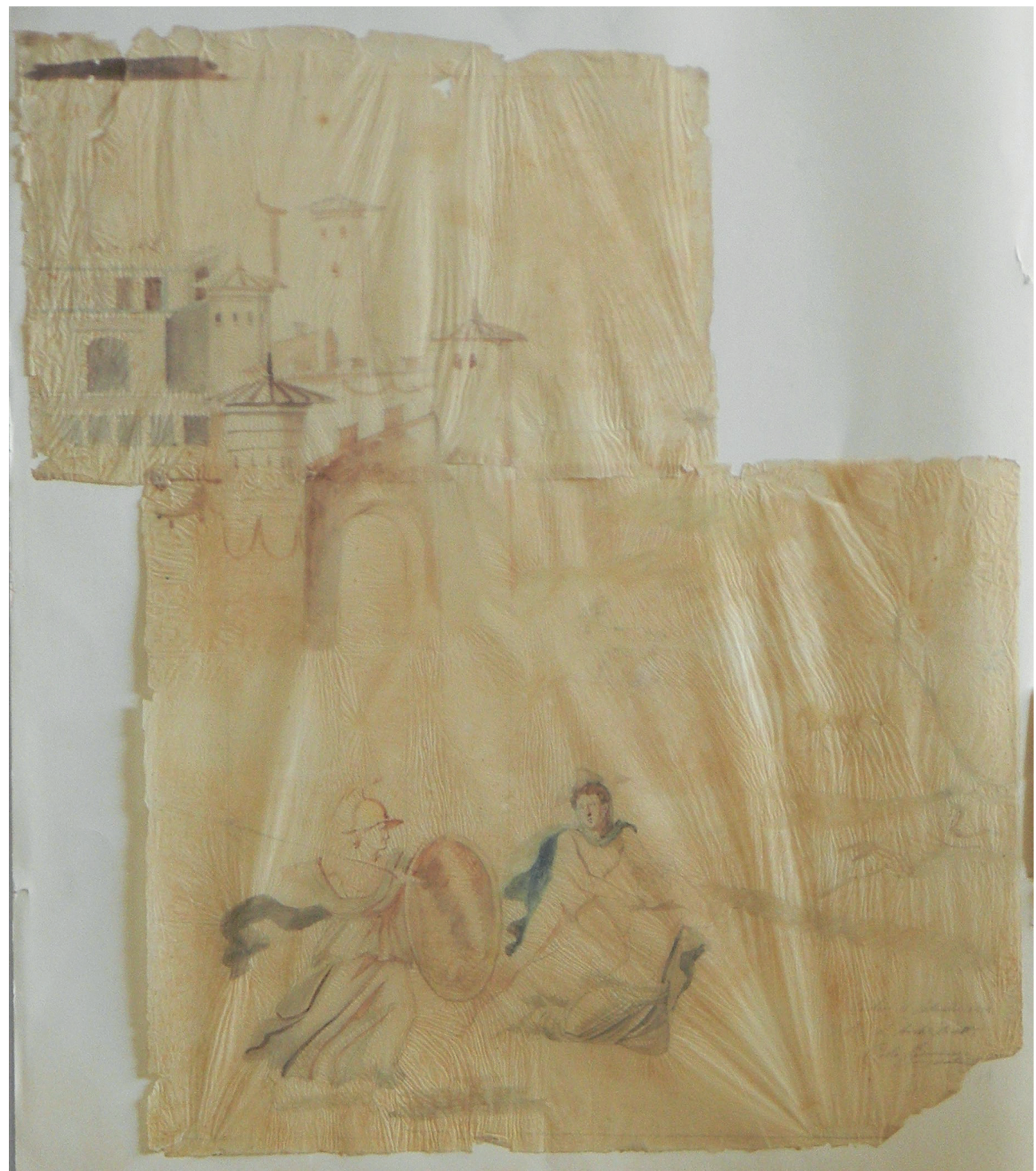

1. Aquarelle réalisée en 1828. Cartella Ercolano RA/10 n. 5530, Archivio disegni della Soprintendenza per $i$ Beni archeologici di Napoli. Avec l'aimable autorisation de la Soprintendenza et du Ministero dei Beni e delle Attività Culturali e del Turismo. 


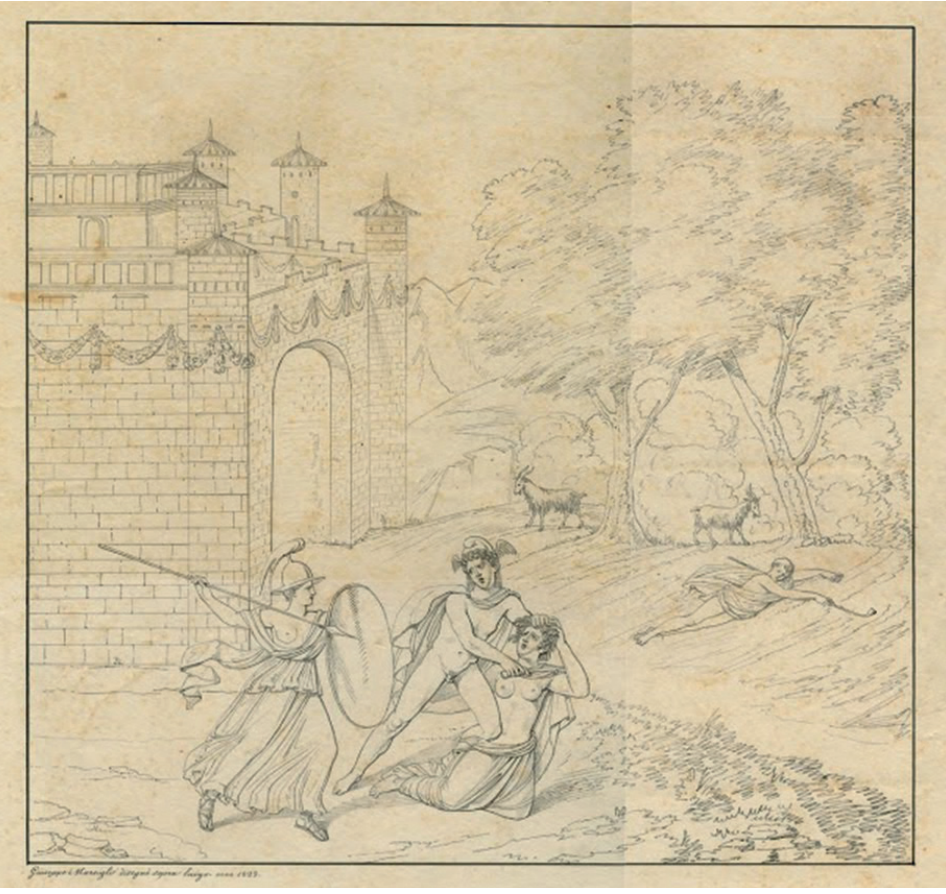

1. Dessin

au crayon exécuté

en 1829.

Cartella Ercolano

RA/10 n. 5530,

Archivio disegni della

Soprintendenza per

i Beni archeologici

di Napoli.

Avec l'aimable

autorisation de

la Soprintendenza

et du Ministero dei

Beni e delle Attività

Culturali e del

Turismo.

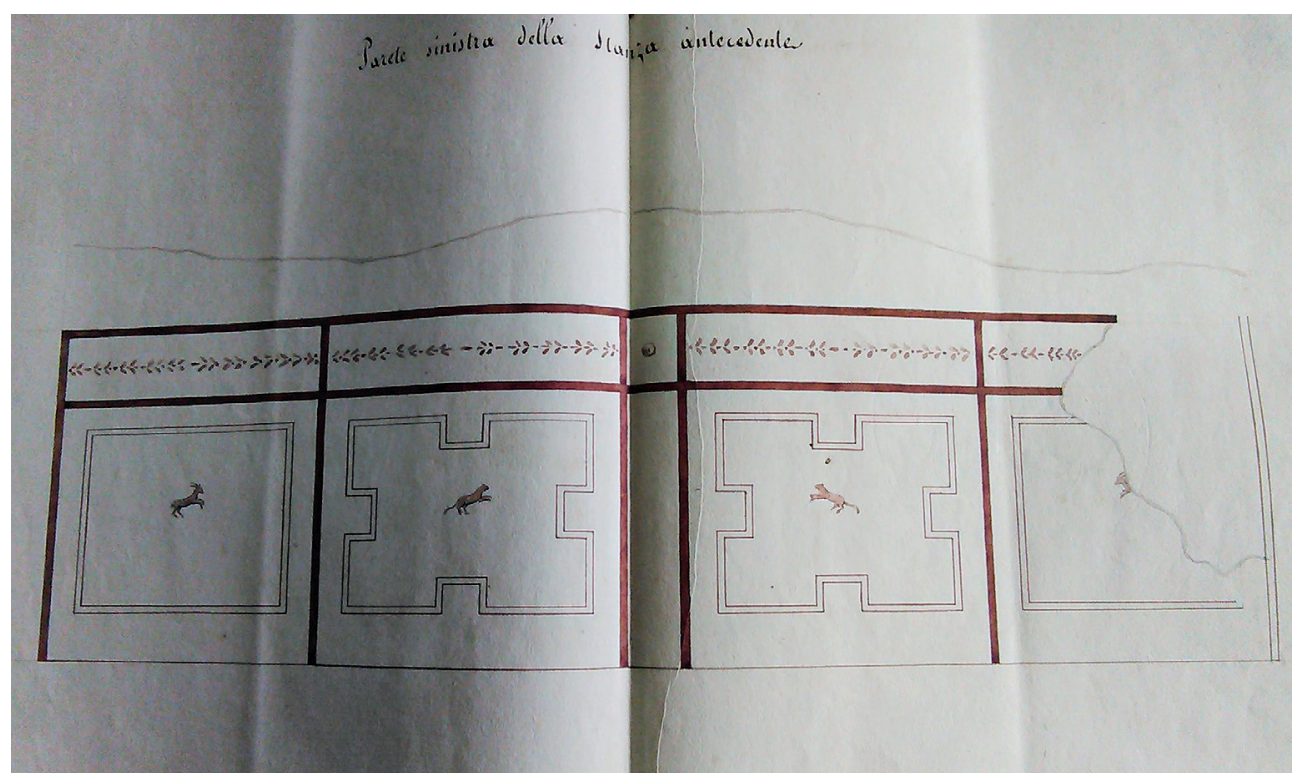

2. Dessin du décor d'une paroi de l'étage supérieur. Archivio storico della Soprintendenza per i Beni archeologici di Napoli ; fasc. VII C9, 3. Avec l'aimable autorisation de la Soprintendenza et du Ministero dei Beni e delle Attività Culturali e del Turismo. 


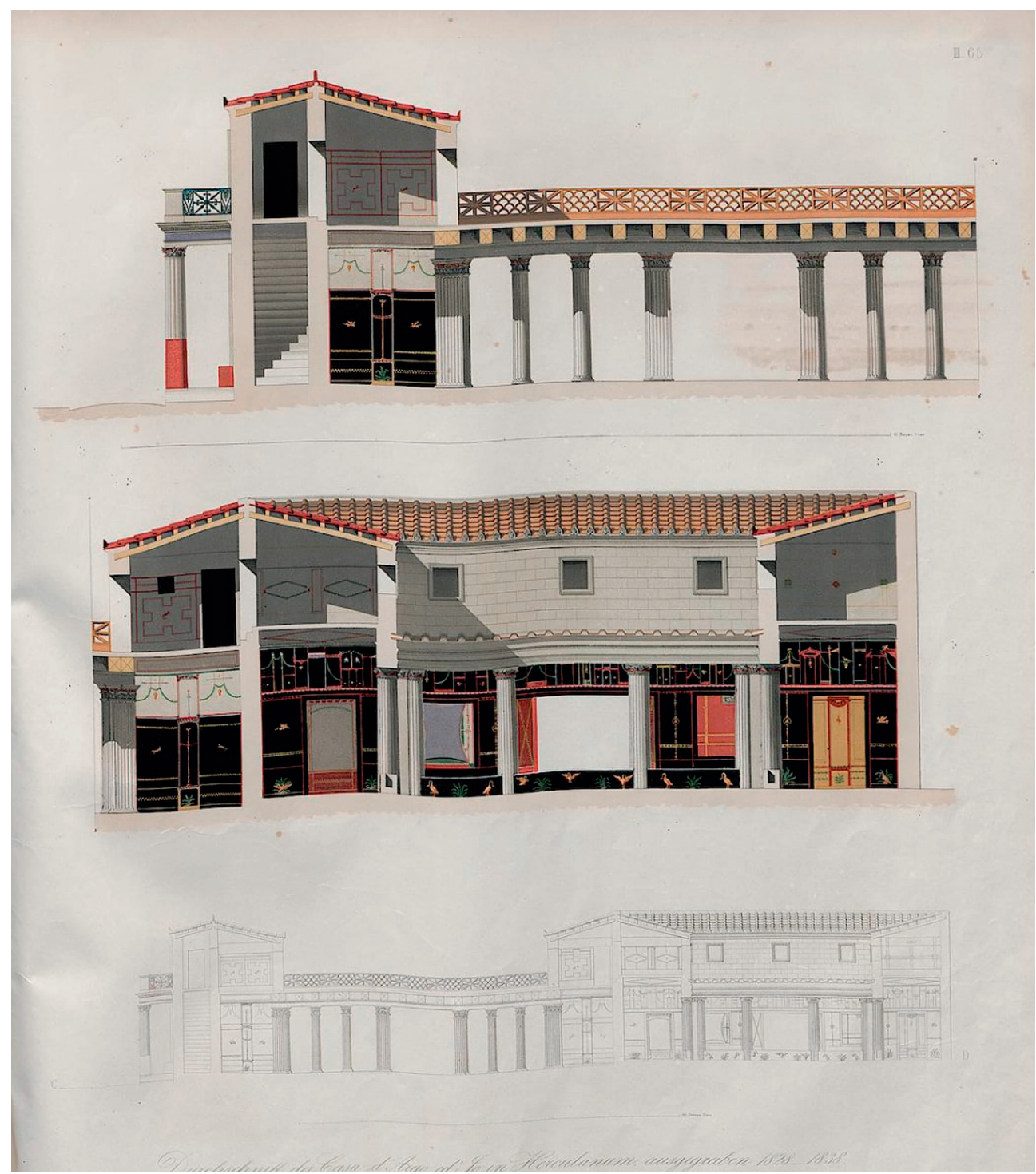

1. Coupe du mur de l'étage supérieur et du rez-de-chaussée. W. Zahn, Les plus beaux ornements, tableau 65 
1. Statuette de Dionysos provenant de « l'atelier du plumbarius »(VI, 12) à Herculanum. D’après T. Budetta - M. PAgano, Legni e bronzi, fig. p. 110.
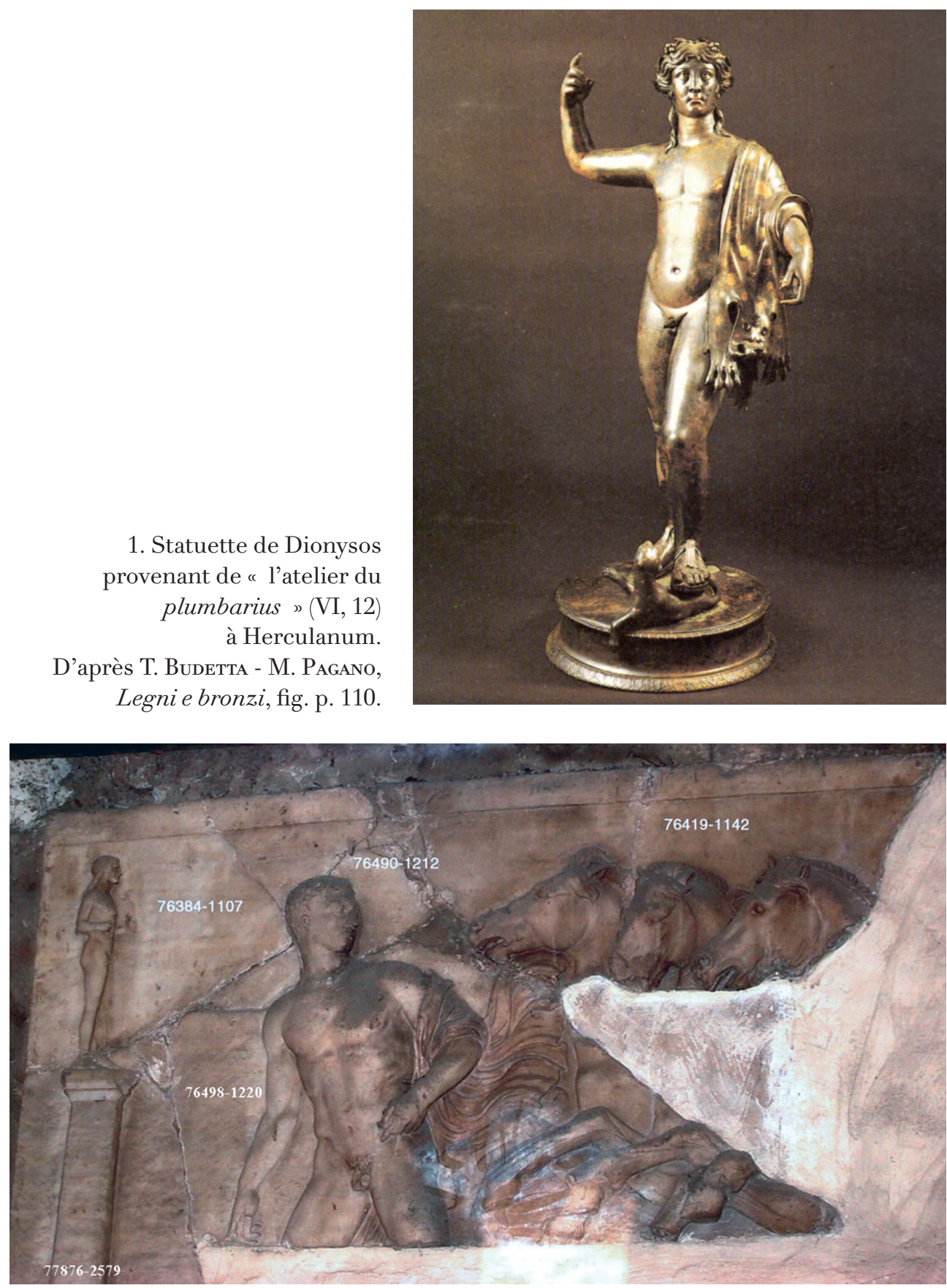

2. Herculanum, Casa del Bel Cortile : les fragments du “ relief A » et leurs numéros d'inventaire. Cliché N. Monteix. 
1. Herculanum, Casa del Bel Cortile : le fragment 1220. Cliché N. Monteix.
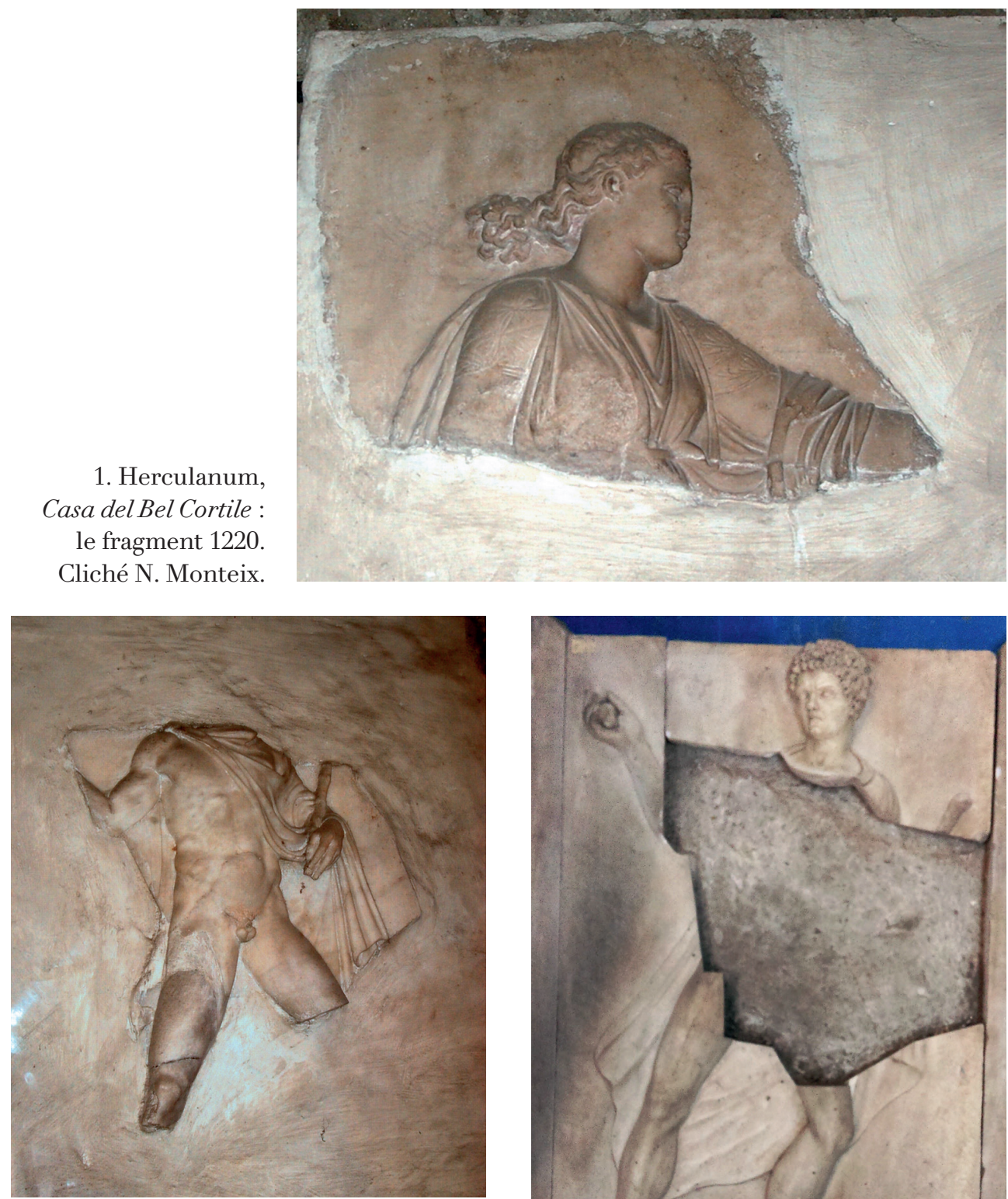

2. Herculanum, Casa del Bel Cortile, “ relief B » : le fragment 1215.

Cliché N. Monteix.

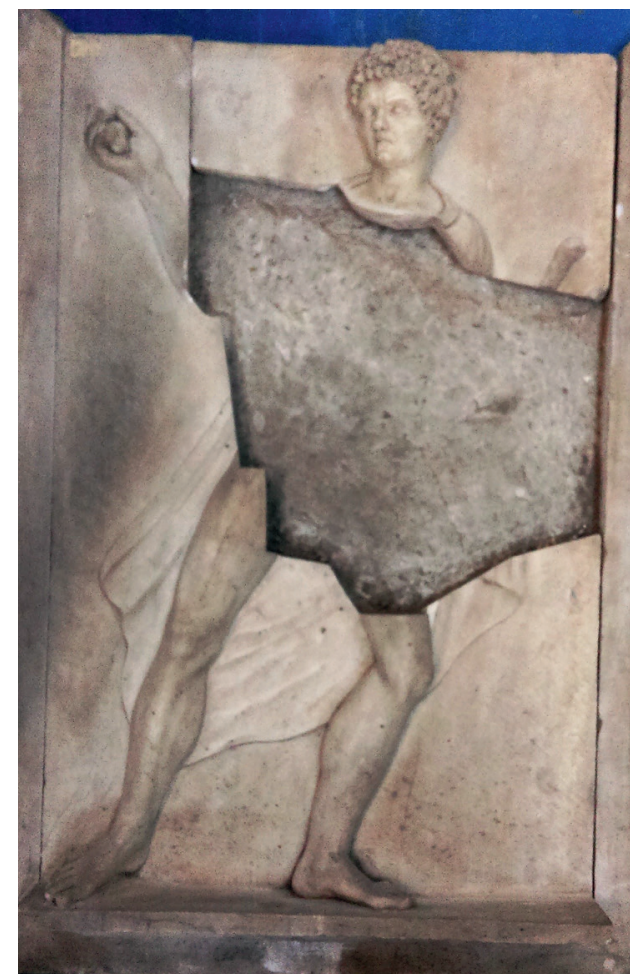

3. Naples, MANN, inv. 6680, relief dérestauré, après prélèvement du fragment appartenant au " relief A »d'Herculanum. Cliché E. Rosso. 

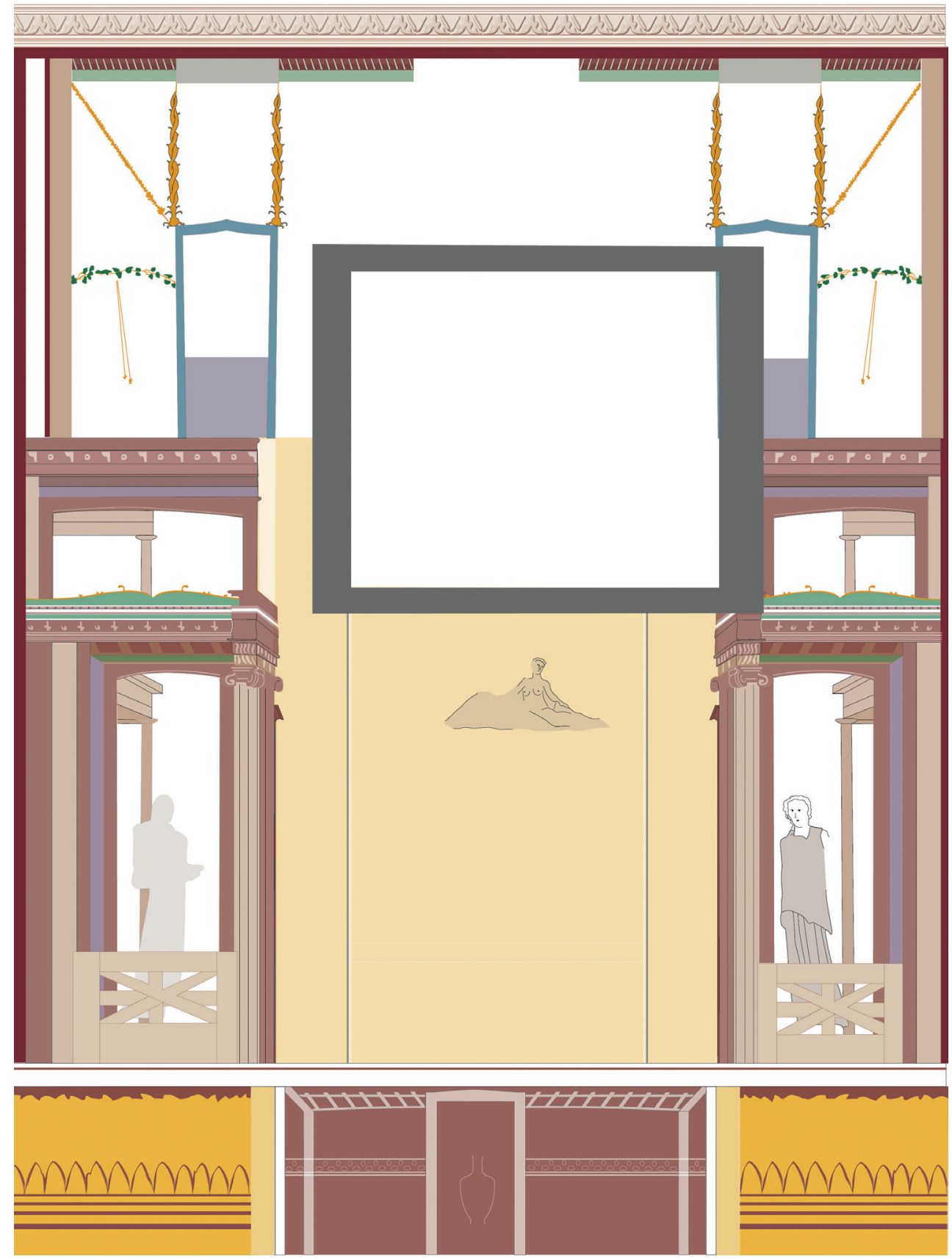

1. Maison de Neptune et Amphitrite, pièce 4, mur est, restitution (infographie M.-L. Maraval). 


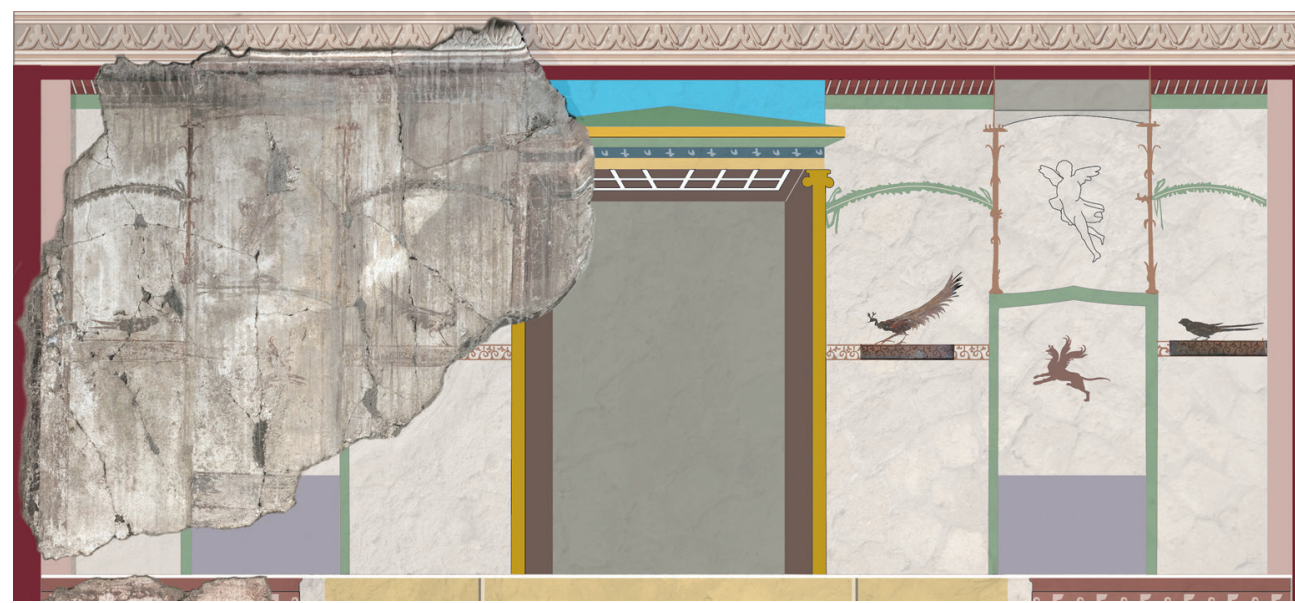

1. Maison de Neptune et Amphitrite, pièce 4, mur sud, zone supérieure, restitution avec les enduits in situ (infographie M.-L. Maraval).

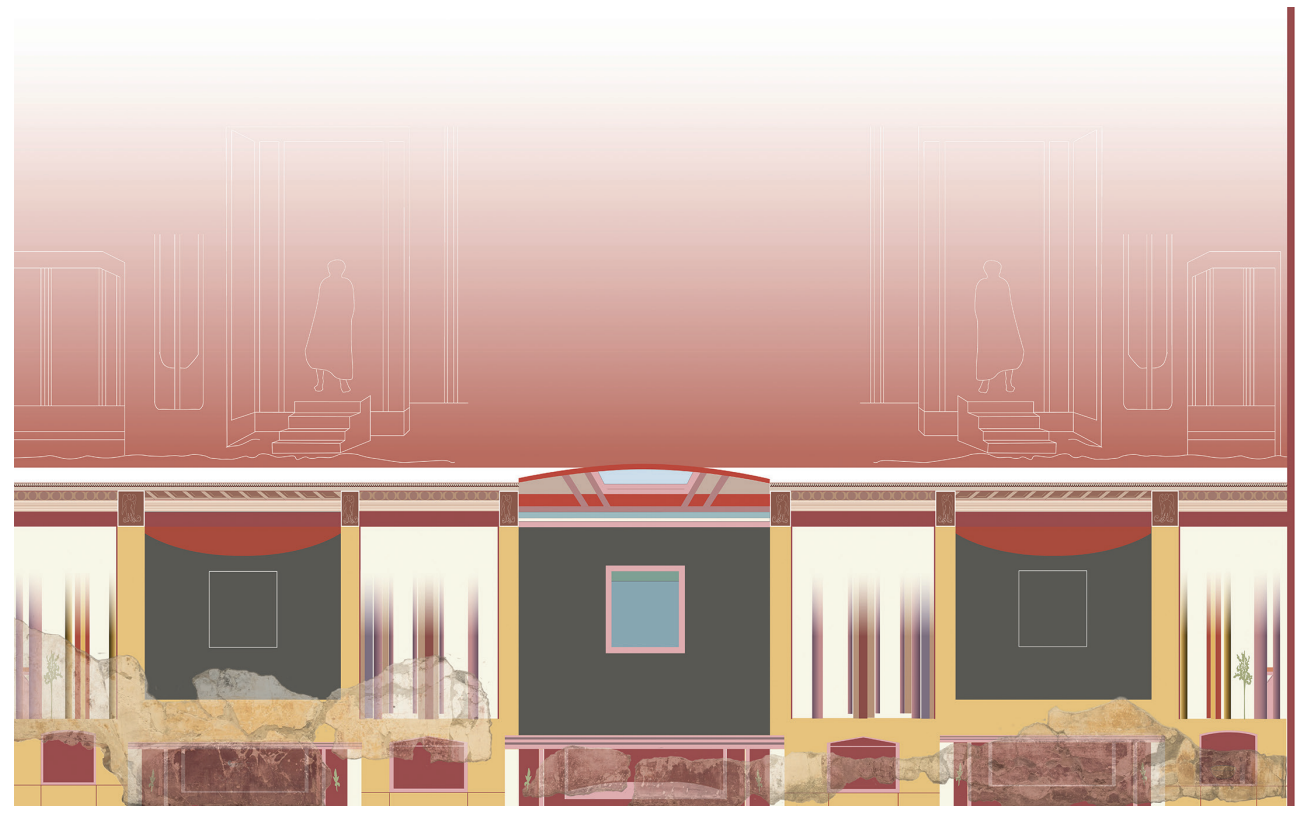

2. Maison de Neptune et Amphitrite, atrium 10, mur nord, restitution (infographie M.-L. Maraval). 


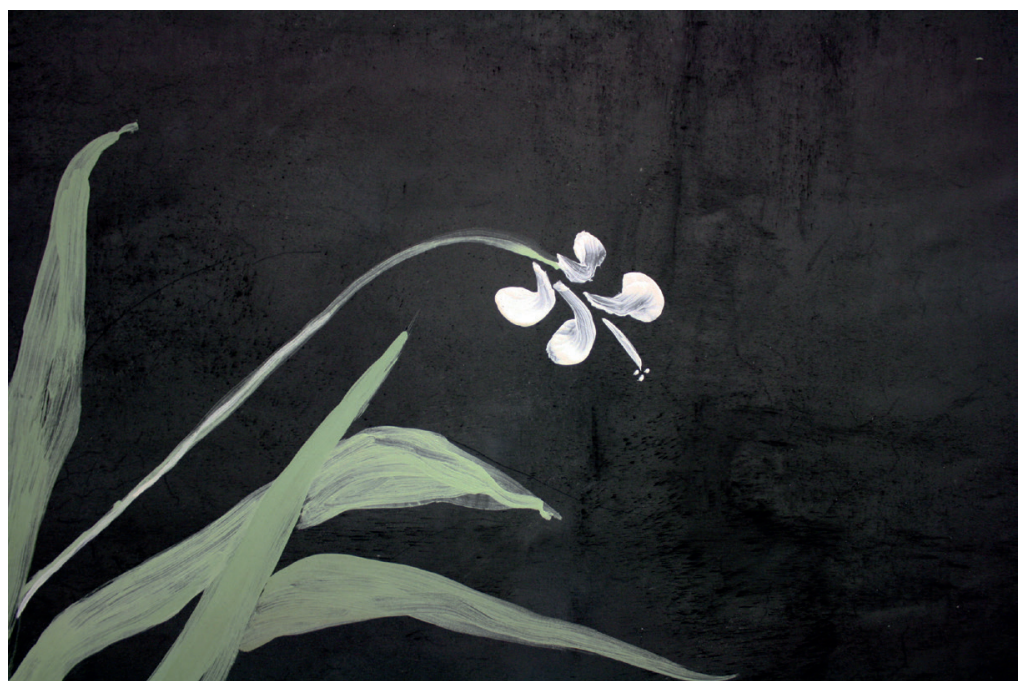

1. Détail du soubassement de la fresque expérimentale réalisée pour l'exposition L'Empire de la Couleur, de Pompéi au Sud des Gaules, Musée Saint-Raymond, Musée des Antiques de Toulouse, 2014-2015. Irrégularité du fond pourtant traité en aplat (fusées, taches...) et transparence de la touche picturale qui laisse voir le fond noir créant de nombreuses nuances de couleurs.

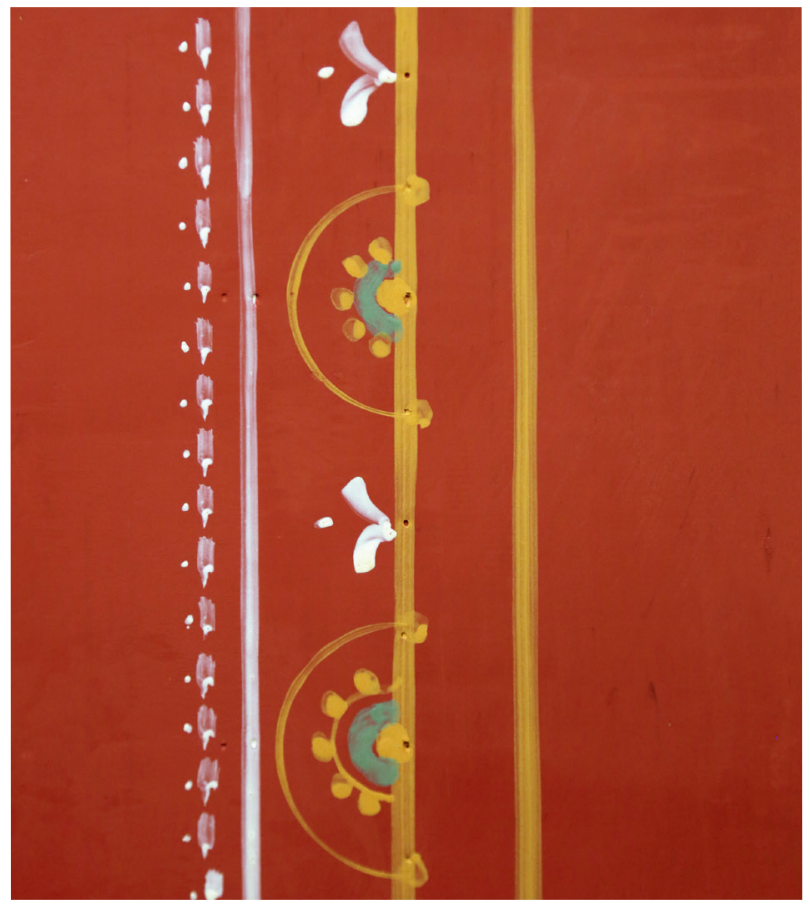

2. Détail d'un panneau

en zone médiane de la fresque expérimentale réalisée pour l'Exposition L'Empire de la Couleur, de Pompéi au Sud des Gaules, Musée Saint-Raymond, Musée des Antiques de Toulouse, 2014-2015. Bordures ajourées sur un fond rouge dont on distingue quelques irrégularités dans le fond ; les motifs répétitifs présentent aussi des irrégularités et des zones de transparence qui laissent voir le fond rouge. 


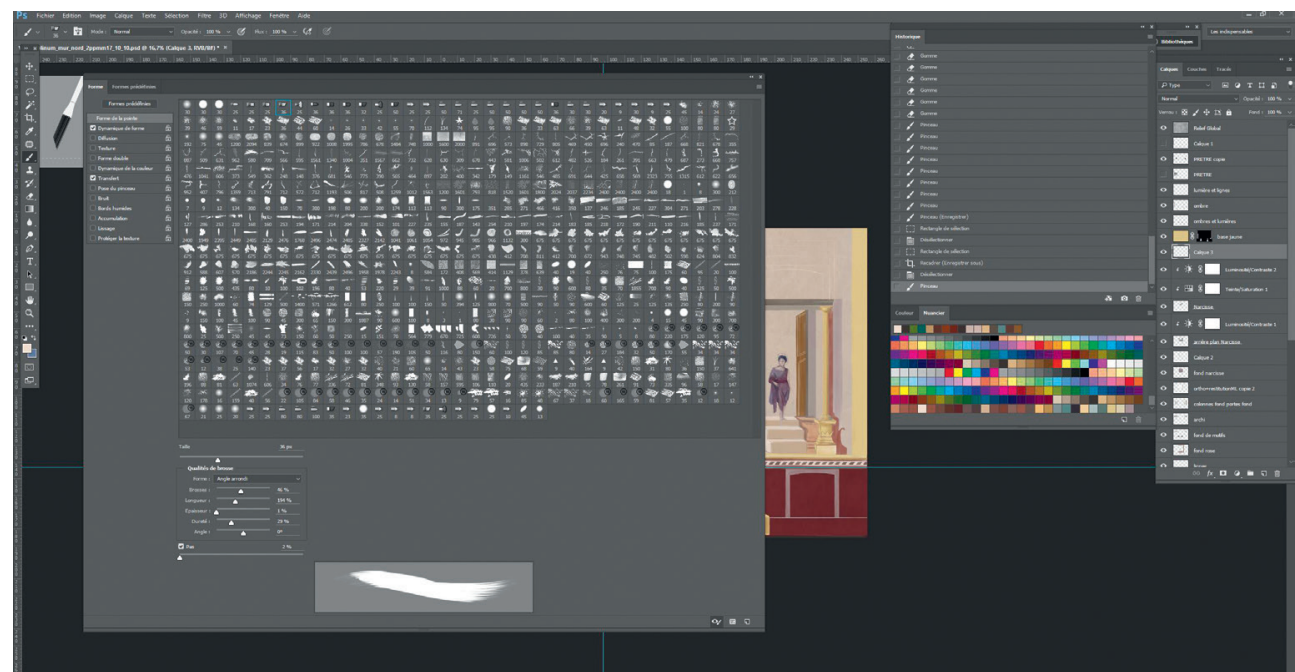

1. Fenêtre des brosses ouvertes dans le logiciel Adobe Photoshop, permettant de voir leur variété de formes et une partie des réglages possibles ; une brosse dynamique est sélectionnée (sa position est visible en haut à gauche). À l'arrière, une partie de la peinture virtuelle et à droite, sous l'historique, la palette des couleurs, puis les calques superposés.

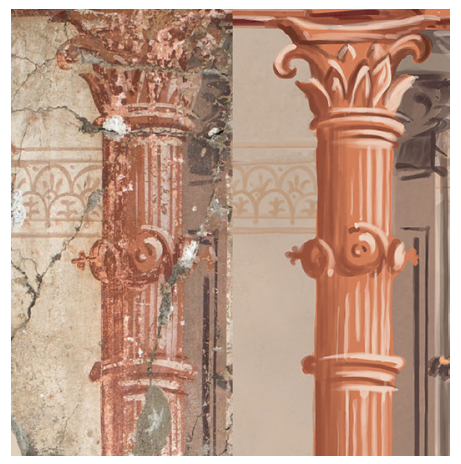

2. « Restauration virtuelle » d'une colonne du triclinium de la Maison de Neptune et Amphitrite à Herculanum : la brosse utilisée pour les touches blanches a permis de garder en transparence le fond lorsqu'elle est appliquée rapidement et au contraire de créer des effets d'accumulation correspondant au levé du pinceau qui produit une zone plus opaque (cf. pl. IX.2).

3. Détail de motifs répétitifs réalisés à main levée sur un fond rouge présentant des irrégularités. Peinture virtuelle du mur sud du triclinium 7 de la Maison de Neptune et Amphitrite à Herculanum.

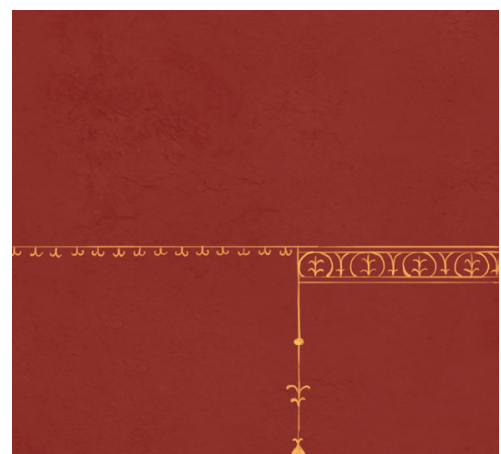




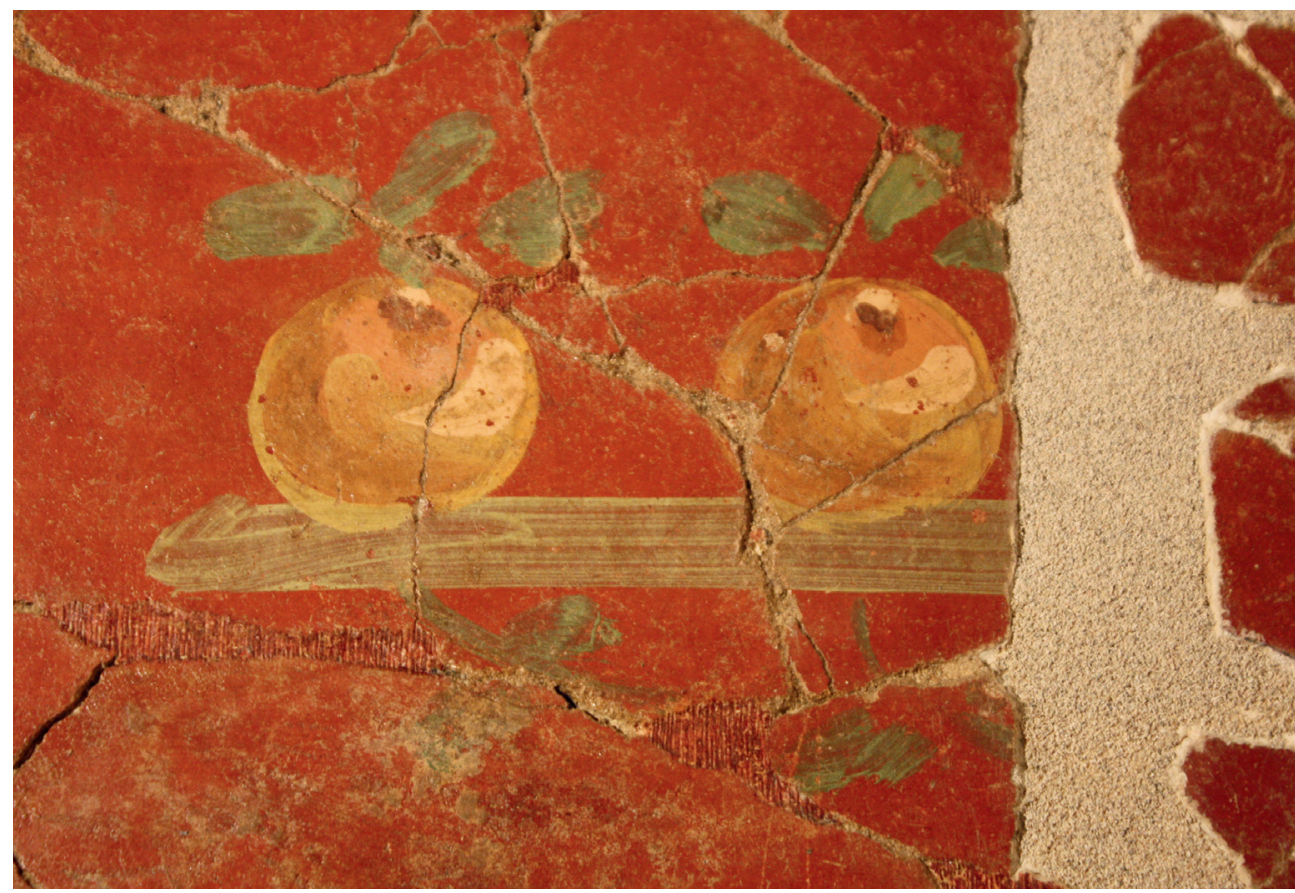

1. Détail d'une peinture provenant du Clos de la Lombarde, Musée de Narbonne. Fruits peints sur fond rouge réalisés à partir de plusieurs couleurs mélangées en amont et juxtaposées de façon à créer un dégradé afin de produire leur rendu sphérique ; le vert des feuilles et du plan laisse voir les effets du pinceau et transparaître la couleur de fond.

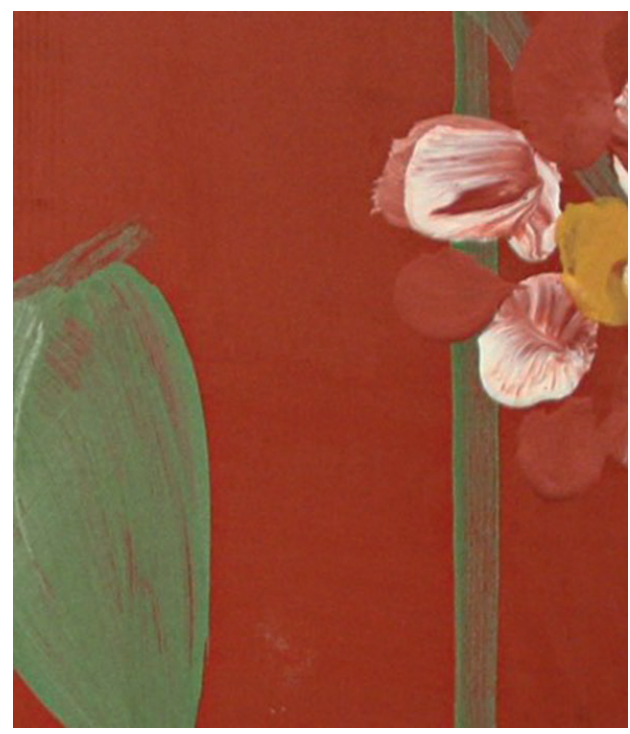

2. Détail d'un essai de peinture

à fresque réalisé en amont de la peinture exposée au Musée Saint-Raymond : le mélange des couleurs pures appliquées directement sur le support pour la réalisation des pétales roses crée un effet pictural très éloigné de celui d'une peinture romaine, contrairement au feuillage vert, où la couleur appliquée uniformément laisse transparaître le fond créant ainsi des variations de tons. 

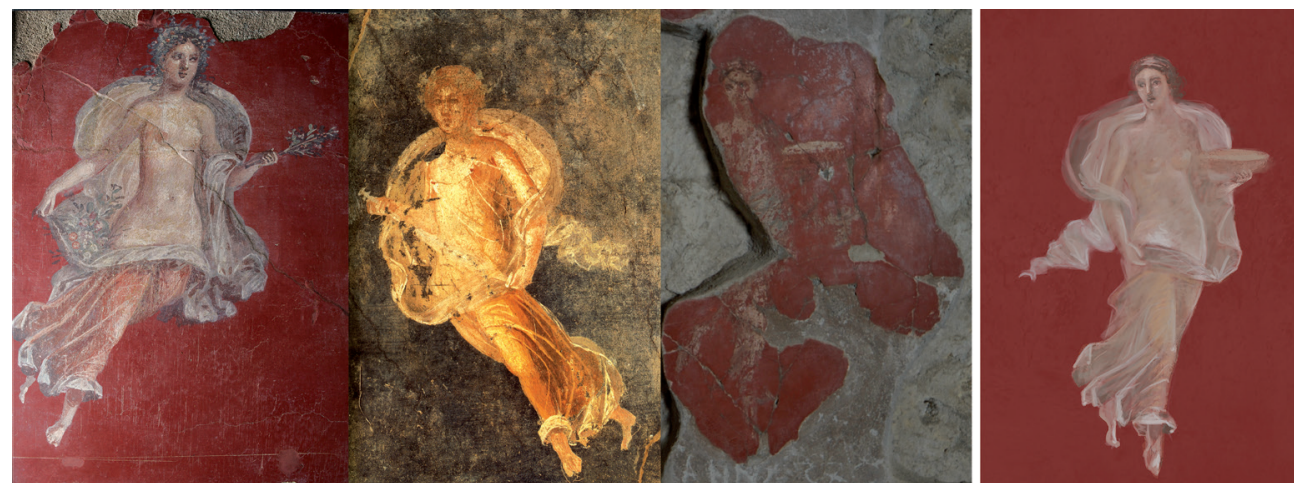

1. Restauration numérique d'une figure volante (à droite) à partir (de gauche à droite) d'une figure provenant de la même salle mais détachée et conservée au MANN ( $\mathrm{n}^{\circ}$ inv. 8835), d'une autre figure volante, sur fond noir, provenant de la maison de Holconius Rufus à Pompéi, et de ses vestiges conservés in situ et très effacés.

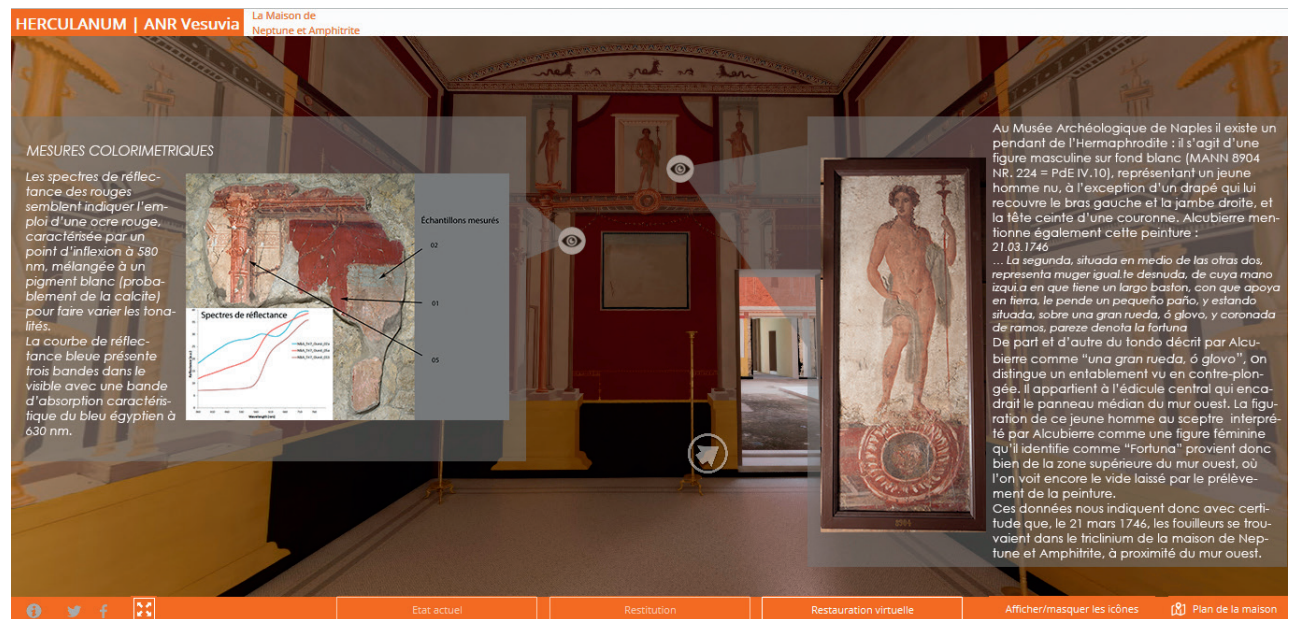

2. Vue du modèle 3D du triclinium 7 de la maison de Neptune et Amphitrite à Herculanum, réalisé par Archéovision-Archéotransfert, avec sa restauration numérique et deux zones cliquables ouvertes pour accéder à des informations complémentaires (ici des mesures colorimétriques et des données sur un panneau conservé au MANN et le contexte de sa découverte) ; au centre un panneau suggéré car aucun indice ne permet d'identifier la scène qui y était représentée. 